\title{
Analytical methods for determination of glutathione and glutathione disulfide in pharmaceuticals and biological fluids
}

\author{
${ }^{1}$ Department of Pharmaceutical Analytical Chemistry, Faculty of Pharmacy, Menofia University, Al Minufiyah 32511, Egypt. \\ https://orcid.org/0000-0003-3934-2326. \\ ${ }^{2}$ School of Chemical Sciences, National Centre for Sensor Research, Dublin City University, Dublin 9, Ireland. \\ https://orcid.org/0000-0002-1945-3574. \\ 3 Dar Al-Fouad Hospital, Naser City, Cairo, Egypt \\ ${ }^{4}$ Department of Pharmaceutical Analytical Chemistry, Faculty of Pharmacy, Tanta University, El-Geish Street, The Med- \\ ical Campus Tanta, Egypt, Phone: +201066698099, Fax: +513-529-5715, E-mail: fotouhrashed@pharm.tanta.edu.eg. \\ https://orcid.org/0000-0003-0847-4035. \\ ${ }^{5}$ Pharmaceutical Services Center, Faculty of Pharmacy, Tanta University, Tanta 31111, Egypt, E-mail: \\ fotouhrashed@pharm.tanta.edu.eg. https://orcid.org/0000-0003-0847-4035.
}

\begin{abstract}
:
Glutathione is a natural tripeptide that plays a major role in different physiological processes in the human body. Determination of glutathione in different body fluids and tissues is essential for early diagnosis and follow-up of various diseases. The assay of glutathione is problematic because of the high polarity, the limited stability, and the aliphatic structure, which lacks the appropriate chromophore for UV detection. A number of methods have been reported for determination of glutathione using different techniques. High-performance liquid chromatography was employed in both reverse phase and hydrophilic interaction modes. Electrochemical methods exploited the redox activity of glutathione to allow for quantification by different electrodes after chemical modification, including glassy carbon, carbon paste, and nanocomposite electrodes. Capillary zone electrophoresis was used with less need for derivatization which makes it simpler, faster, and more economic. A number of nanosensors and probes have been developed to assay glutathione in biological fluids using semiconductor nanoparticles, quantum dots, genetically engineered green fluorescent probes, and new derivatives of known dye classes. This work is an updated review of the methods of analysis of glutathione and glutathione disulfide in pharmaceuticals and biological fluids with more emphasis on the technical problems and the assay artifacts.
\end{abstract}

Keywords: biological fluids, glutathione, glutathione disulfide, pharmaceuticals, review

DOI: $10.1515 /$ revac-2019-0019

Received: October 16, 2019; Accepted: December 11, 2019

\section{Abbreviations}

AF Acetylferrocene

ARS Alizarin red S

AuNPs Gold nanoparticles

BCB 4,5-bis(4-Chloroanilino)-1,2-benzendiol

BCPB 1-Benzyl-2-chloropyridinium bromide

BODIPY Boron dipyrromethene

BODIPY Fl-C $_{1}$-IA N-(4,4-Difluoro-5,7-dimethyl-4-bora-3a,4a-diaza-s-indacene-3-yl)methyl)iodoacetamide

BSA Bovine serum albumin

CAF Polycaffeic acid

CDD Cyanine derivative dye 
CE Capillary electrophoresis

CMQT 2-Chloro-1-methylquinolinium tetrafluoroborate

CNBF 4-Chloro-3,5-dinitrobenzotrifluoride

CNTs Carbon nanotubes

CPE Carbon paste electrodes

CQDs Carbon quantum dots

CT Charge transfer

CV Cyclic voltammetry

Cys L-Cysteine

CZE Capillary zone electrophoresis

DABCYL 4-(4-Dimethylaminophenylazo) benzoic acid

DED 9,10-Dihydro-9,10-ethanoanthracene-11,12-dicarboximido)-4-ethylbenzene-1,2-diol

DHB (E)-3-((2-(2,4-Dinitrophenyl)hydrazono)-methyl)benzene-1,2-diol

DHCA 3, 4-Dihydroxy-cinnamic acid

DHPB N-(3,4-Dihydroxyphenethyl)-3,5-dinitrobenzamide

DMBQ 8,9-Dihydroxy-7-methyl-12H-benzothiazolo[2,3-b]quinazolin-12-one

DMF Dimethyl formamide

DNBSQ Dinitrobenzenesulfonyl coumarin

DNFB 1-Fluoro-2,4-dinitrobenzene dioxane

DPV Differential pulse voltammetry

DTE 1,4-Dithioerythritol

EF Ethynylferrocene

EOF Electro-osmotic flow

ESIPT Excited-state intramolecular proton transfer

FRET Förster resonance energy transfer

GCE Glassy carbon electrodes

GNS@rGO Gold nanostar@reduced graphene oxide composite

GSH Glutathione

GSSG Glutathione disulfide

HCys Homocysteine

HEPES (4-(2-Hydroxyethyl)-1-piperazineethanesulfonic acid)

HILIC Hydrophilic interaction LC

IPC Ion pair chromatography

ISPT Isoprenaline

LC Liquid chromatography

LIF Laser-induced fluorescence

LSPR Localized surface plasmon resonance

LSV Linear sweep voltammetry

MCE Microchip capillary electrophoresis

MCOP Metal-organic coordination polymer

MLCs Metal-ligand complexes

MOPS 3-(N-morpholino)propanesulfonic acid

NAC N-acetylcysteine

NACET N-acetylcysteine ethyl ester

NADH Nicotinamide adenine dinucleotide 
NBD-F 4-Fluoro-7-nitrobenzofurazan

NCL Native chemical ligation

NCs Nanoclusters

NDA Naphthalene-2,3-dicarboxaldehyde

NDS N-(4-methyl-2-oxo-2H-chromen-7-yl)-2,4-dinitrobenzenesulfonamide

NEM N-ethylmaleimide

NHPDA N-(4-hydroxyphenyl)-3,5-dinitrobenzamide

$\mathrm{NiO}$ Nickel oxide

NiONPs Nickel oxide nanoparticles

NR Not reported

OPA $o$-Phthaldialdehyde

-AP $p$-Aminophenol

PDDAC Poly(diallyldimethylammonium chloride)

PET Photo-induced electron transfer

QDL Quantum dot conjugated dopamine ligand

QDs Quantum dots

RPLC Reversed phase LC

SBD-BF Ammonium 5-bromo-7-fluorobenzo-2-oxa-1,3-diazole-4-sulfonate

SBD-F Ammonium 7-fluorobenzo-2-oxa-1,3-diazole-4-sulfonate

SERS Surface-enhanced Raman spectroscopy

SPE Screen-printed electrodes

SWV Square wave voltammetry

TAA Thioacetic acid

TCA Trichloroacetic acid

TGA Thioglycolic acid

t-ITP Transient isotachophoresis

TMPAB-I 1,3,5,7-Tetramethyl-8-phenyl-(4-iodoacetamido)difluoroboradiaza-s-indacene (TMPAB-I).

\section{Introduction}

Glutathione (GSH) is one of the most abundant antioxidants in living organisms (Amir \& Ghobadi, 2016). Glutathione is biosynthesized in human (Zhang \& Rinna, 2009), animal (Chen et al., 2013), plant (Liedschulte et al., 2010), and microbial cells such as fungi (Pradhan et al., 2015) and bacteria (Dalle-Donne et al., 2009). The chemical name of GSH is -L-glutamyl-L-cysteinylglycine. This biological tripeptide plays vital roles in various physiological processes such as DNA synthesis (Benhar et al., 2016; Xue et al., 2016), protein synthesis (Couto, Wood \& Barber, 2016; Giustarini et al., 2017), cell membrane stabilization (Lee et al. 2014a; 2014b; 2014c; Sabiu, O'Neill \& Ashafa, 2016), amino acid transportation (Crawford \& Weerapana, 2016; Shishido et al., 2017), and xenobiotic detoxification (Balabanova et al., 2018; Berenbaum \& Johnson, 2015; Faber et al., 2019). Glutathione acts as an electron donor through reduction of the thiol group in the cysteine moiety and the formation of the oxidized disulfide form (GSSG) (Alisik, Neselioglu \& Erel, 2019). After that, the enzyme, GSH reductase, converts GSSG back to GSH using nicotinamide adenine dinucleotide phosphate hydrogen (NADPH) as a cofactor. Thus, the molar ratio of GSH/GSSG is an important indicator of the cellular oxidative stress. The low GSH/GSSG ratio is an important biomarker in a number of human disorders such as cataract, cancer, parkinsonism, diabetes, renal failure, pneumonia, Alzheimer's disease, lead toxicity, cystic fibrosis, dystrophic skin fibroblasts, neurodegenerative disorders, and liver and human immunodeficiency virus infections.

Determination of GSH in biological fluids is challenging because of the following reasons: (i) GSH can be easily autoxidized to GSSG, which results in a lower GSH/GSSG ratio; (ii) the proteolytic degradation of both GSH and GSSG by $\gamma$-glutamyl transferase; (iii) the lack of good UV/Vis chromophore or native fluorophore, 
which makes direct measurement not quite practical; and (iv) the high polarity, which hinders liquid extraction by common organic solvents.

The number of publications on GSH in different research disciplines is increasing every year (Figure 1). The different methods used for determination of GSH and GSSG in biological samples have been already reviewed in early 2009 (Monostori et al., 2009). This work is an update of Monostori and colleagues' (2009) review covering the period (2009 to mid-2019), giving more emphasis on the technical problems and the assay artifacts.
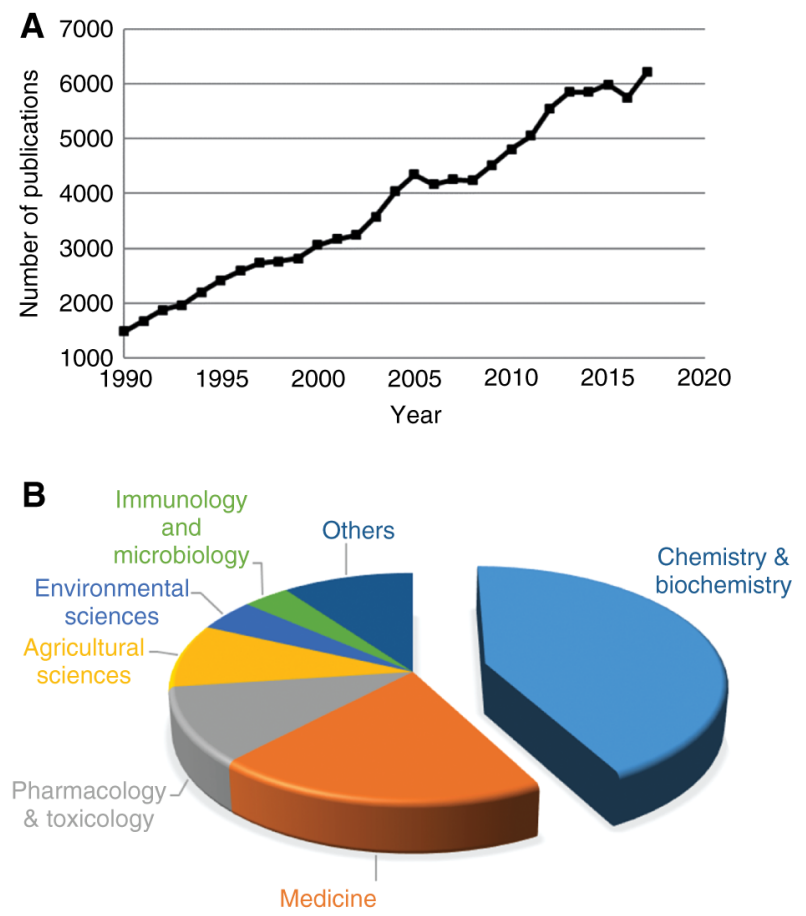

Figure 1: (A) Number of publications using glutathione as a keyword since 1990. (B) Number of glutathione publications in different research areas in the last three decades.

\section{Discussion}

\subsection{Liquid chromatographic methods}

Liquid chromatography (LC) has been extensively used in pharmaceutical (Siddiqui, AlOthman \& Rahman, 2017) and biomedical analysis of drugs (Mansour \& Danielson, 2012; Mansour, Wei \& Danielson, 2013), metabolites (Mansour \& Danielson, 2012; Schleicher et al., 2016), impurities (Ragab \& El-Kimary, 2017; Mabrouk et al., 2019c), biomarkers (Liu et al., 2015), and xenobiotics (Asimakopoulos et al., 2016) for decades. Among the common chromatographic modes of LC, reversed phase LC (RPLC), ion pair chromatography (IPC), and hydrophilic interaction LC (HILIC) have been recently reported for determination of GSH (Table 1). In this section, the recent chromatographic modes and the future trends in GSH determination by high-performance LC (HPLC) are discussed.

\subsubsection{Chromatographic modes of CSH determination}

A few RPLC methods have been reported for determination of GSH in pharmaceutical dosage forms. Sutariya, Wehrung, and Geldenhuys (2012) developed a method for quantification of GSH in nanoparticle solutions using direct UV detection at $215 \mathrm{~nm}$. The mobile phase was acetonitrile: water ( $\mathrm{pH} 7.0)$ (50:50, vol/vol) at a flow rate of $1 \mathrm{ml} / \mathrm{min}$. The method was simple and did not require derivatization, but the retention time was very small ( $<1 \mathrm{~min}$ ). The retention factor was not calculated, and the blank chromatogram was not shown to check if the analyte did not elute in the void volume. The authors evaluated the method specificity by visual inspection of the GSH peak, which could be misleading especially if both the analyte and the interfering substances are unretained. 


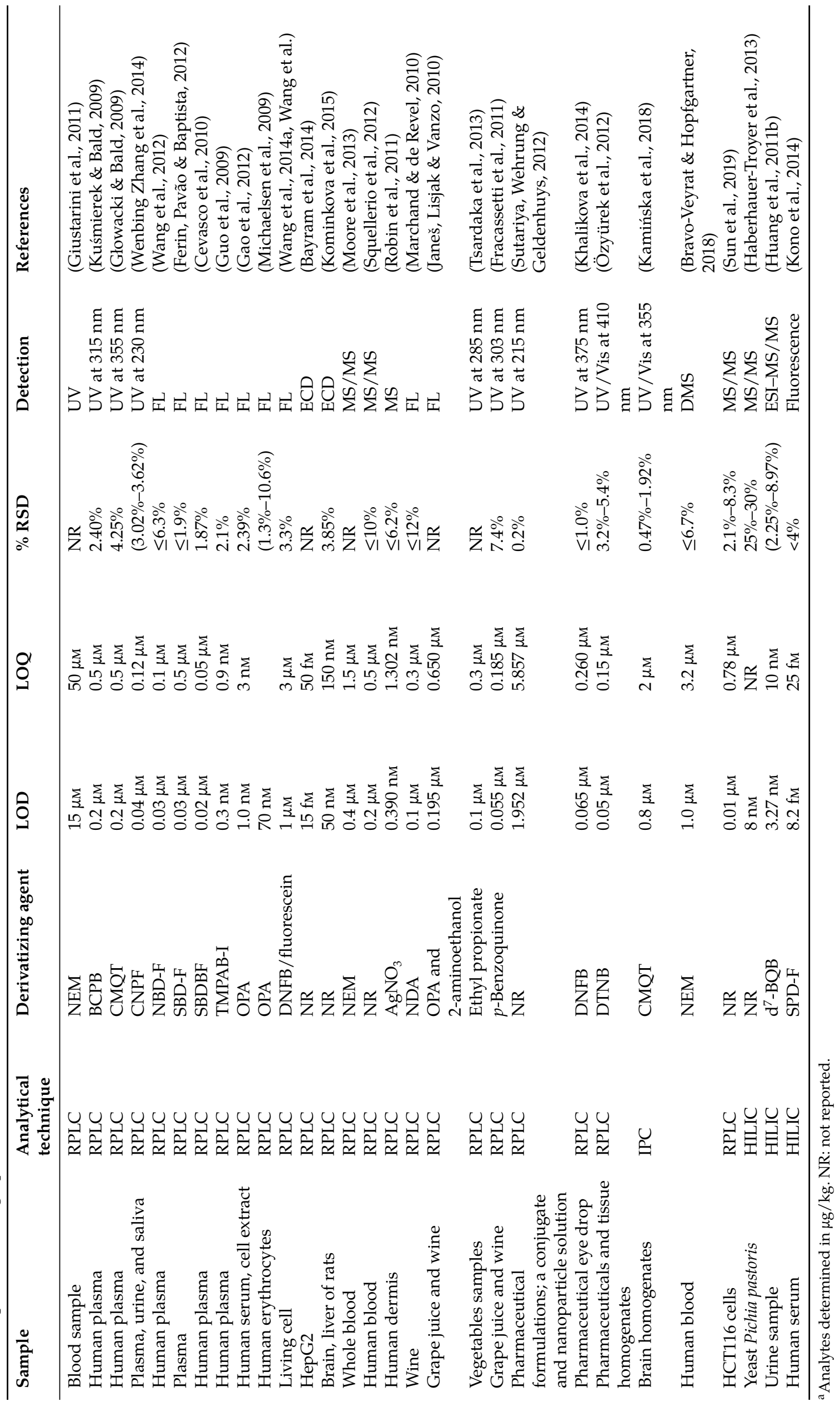


A more sensitive method was developed by Khalikova et al. (2014) for simultaneous measurement of GSH, taurine, and carnosine in eye drops using precolumn derivatization. The derivatization reaction was performed using 10\% 1-fluoro-2,4-dinitrobenzene dioxane (DNFB) in $50 \mathrm{~mm}$ sodium tetraborate, with vigorous shaking for $0.5 \mathrm{~min}$ and heating for $1 \mathrm{~h}$ at $40{ }^{\circ} \mathrm{C}$, before dilution with acidified methanol and filtration. The separation was performed through gradient elution of two mobile phases: A was $50 \mathrm{~mm}$ triethylamine in acetonitrile, and B was $30 \mathrm{~mm}$ trimethylamine ( $\mathrm{pH}$ adjusted to 2.5 with glacial acetic acid). Despite the acceptable accuracy and precision, the method was time consuming and sophisticated for dosage form analysis.

A simpler approach was proposed by Özyürek et al. (2012) using postcolumn derivatization by DTNB [dissolved in a solution of $0.5 \%$ methyl- $\beta$-cyclodextrin ( $\mathrm{wt} / \mathrm{vol}$ ), $\mathrm{pH} 8$ ]. The yellow colored reagent was monitored at $410 \mathrm{~nm}$, and the elution of the thiol analyte was associated with a negative peak. The analytes could be also monitored at $205 \mathrm{~nm}$ where positive peaks were observed, but at the expense of the method sensitivity. These procedures were applicable for the determination of other biothiols, including L-cysteine (Cys), N-acetylcysteine (NAC), homocysteine (HCys), and 1,4-dithioerythritol (DTE). The method was very selective for determination of biothiols in presence of nonthiol amino acids and polyphenolic antioxidants. The long retention time observed for GSH (4.3 min) was due to the gradient elution using a mobile phase consisting of methanol and 2.5 mm phosphate buffer ( $\mathrm{pH} 2.85)$.

Determination of GSH in biological fluids is more challenging because of the matrix effect and the expected interference. UV is the most common detector in HPLC and does not require frequent maintenance procedures (Kuśmierek \& Bald, 2009). A few recent methods were reported for determination of GSH in biological fluids using RPLC/UV detection. Giustarini et al. (2011) developed a method for determination of GSH in human plasma using N-ethylmaleimide (NEM) as a derivatizing agent and 12\% trichloroacetic acid (TCA) for protein precipitation. The NEM reaction with GSH was fast $(1 \mathrm{~min})$ and did not require heating or catalysis, and the GSH conjugate with NEM was stable against oxidation. However, the method suffered from the following limitations: the Limit of Detection (LOD) is poor compared with the other reported RPLC methods; two symmetric peaks of equal intensity were obtained for GSH-NEM, which complicates the separation; and the proposed method was not suitable for determination of oxidized GSH. These limitations were solved by Kuśmierek and Bald (2009), who added tris(2-carboxyethyl)phosphine hydrochloride as a reducing agent before derivatization with 1-benzyl-2-chloropyridinium bromide (BCPB). The method was significantly more sensitive (LOD = $0.2 \mu \mathrm{M})$ and able to determine total plasma thiols, but the linear dynamic range was small $(0.5-40 \mu \mathrm{M})$. Similar sensitivity was attained by using 2-chloro-1-methylquinolinium tetrafluoroborate (CMQT) as a chromogenic agent and fully automating the reaction before inline sample deproteinization through dialysis (Głowacki \& Bald, 2009). Higher sensitivity was observed when 4-chloro-3,5-dinitrobenzotrifluoride (CNBF) was employed for derivatization with a wider dynamic range of $0.15-500 \mu \mathrm{M}$. However, longer reaction time was required (10 min) compared with CMQT (Kamińska et al., 2018; Zhang et al., 2014).

More methods were reported for determination of GSH by RPLC/FL detection. Halogenated benzofurans were commonly used as fluorogenic agents for GSH derivatization. These reagents were selective for thiol compounds and did not have native fluorescence, which decreased the risk of interference from excess reagents. In addition, the reaction products were highly fluorescent and appreciably retained in RPLC. Ferin, Pavão, and Baptista (2012) used ammonium 7-fluorobenzo-2-oxa-1,3-diazole-4-sulfonate (SBD-F) as a derivatizing agent for determination of GSH in plasma after reduction with tri-n-butylphosphine, but the reaction time was long (60 $\mathrm{min})$, and the reaction temperature was high $\left(60^{\circ} \mathrm{C}\right)$. To accelerate the reaction, a bromo-derivative of SBD-F was used (ammonium 5-bromo-7-fluorobenzo-2-oxa-1,3-diazole-4-sulfonate; SBD-BF) (Cevasco et al., 2010). The electron withdrawing group in SBD-BF made it more reactive and shortened the reaction time (30 $\mathrm{min})$. Milder reaction conditions $\left(10 \mathrm{~min}\right.$ at $30^{\circ} \mathrm{C}$ ) were attained by Wang et al., via using 4-fluoro-7nitrobenzofurazan (NBD-F). Despite the favorable reaction conditions, the product was unstable as evidenced by the decrease in peak area with increased column temperature or storage at $-20{ }^{\circ} \mathrm{C}$ for more than $72 \mathrm{~h}$ (Wang et al., 2012).

A low concentration in the nM level was reached by Michaelsen et al. (2009) through using ophthaldialdehyde (OPA) as a derivatizing agent and the thiol-reducing agent: NAC ethyl ester (NACET). Although NACET is a strong and specific disulfide-reducing agent, OPA is nonspecific for thiol groups, and GSH-OPA is unstable, which affects the method accuracy and precision (Wang et al., 2012). The most sensitive RPLC/FL method was presented by Guo et al. (2009) by using 1,3,5,7-tetramethyl-8-phenyl-(4iodoacetamido)difluoroboradiaza-s-indacene (TMPAB-I). A limit of detection as low as 0.3 nM was achieved for GSH using an isocratic method and a precolumn derivatization reaction at $45^{\circ} \mathrm{C}$ for 20 min. Regardless of the impressively high sensitivity, the difference between the excitation $(500 \mathrm{~nm})$ and the emission wavelengths (510) is small, which increases the risk of self-excitation.

Two methods were reported for electrochemical detection of GSH in RPLC. Both methods were highly sensitive with no need for chemical derivatization. Kominkova et al. (2015) used amperometric detection at 900-mV potential for determination of GSH in rats' brain and liver. The analysis time was 20 min, and gradient elution 
was required. A simpler, faster, and more sensitive method was developed by Bayram et al. (2014) for separation of ascorbic acid, uric acid, GSH, and GSSG within 5 min using coulometric electrochemical detection. The developed method was successfully used for assay of GSH in hepatocytes with a sensitivity level up to $15 \mathrm{fm}$.

Mass spectrometry (MS) is the most sensitive detection mode in RPLC. Besides, LC-MS is highly accurate, precise, and specific especially when more than one fragment is being monitored. Moreover, the derivatization step is nonessential because even nonchromophoric analytes and highly diluted samples can be assayed directly. Moore et al. (2013) determined GSH and GSSG in human blood by LC-MS/MS using a mixture of sulfosalicylic acid and NEM for deproteinization and derivatization, respectively. A Hypercarb column (Thermo Fisher Scientific, Rockford, IL, USA) was used for separation to improve the retention of such polar analytes and to focus the early eluting peak of ion-suppressing salts. Higher sensitivity was obtained by Squellerio et al. (2012) without using a derivatizing agent in a short analysis time ( $<6 \mathrm{~min})$. The detection limit could be improved further by utilizing $\mathrm{AgNO}_{3}$ as a pot-column reagent (Robin et al., 2011). Five-fold increases in response were obtained when silver nitrate was used, with a doublet peak due to the presence of two equally abundant isotopes of silver: ${ }^{107} \mathrm{Ag}$ and ${ }^{109} \mathrm{Ag}$.

Hydrophilic interaction LC is a special chromatographic mode that employs polar stationary phases (similar to normal phase) with aqueous mobile phases (similar to reversed phase) to separate highly polar and ionic compounds (similar to ion exchange chromatography). Due to the high polarity of GSH, HILIC was a suitable alternative to the conventional reversed phase mode. Haberhauer-Troyer et al. used HPLC-MS/MS for determination of GSH/GSSG ratios in Pichia pastoris yeast using HILIC (Haberhauer-Troyer et al., 2013). The method did not require chemical derivatization, but the uncertainty was in the range $25-30 \%$. A more sensitive and precise method was developed using $\mathrm{d}^{7}$ - $\omega$-bromoacetonylquinolinium bromide ( $\mathrm{d}^{7}$-BQB) (Huang et al., 2011a, Huang et al., 2011b). The sensitivity could be further improved using SBD-F as a derivatizing agent and fluorescence as a detector (Kono et al., 2014). Limits of quantitation in the $\mathrm{fm}_{\mathrm{M}}$ levels could be attained for GSH and other endogenous thiols in human serum. Compared with RPLC methods for GSH determination, HILIC has two advantages: it can retain polar analytes such as GSH without derivatization, and the high organic content of the mobile phase facilitates the desolvation step in MS detection.

\subsubsection{Trends in $\mathrm{LC}$ for CSH determination}

Analysis of GSH at point of care is of potential need to monitor oxidative stress and pathophysiological changes in endogenous antioxidants. Although HPLC is highly reliable, the bulky size and weight of the instrument hinder its on-site applications. For this reason, instrument miniaturization is required to increase portability and allow real-time analysis of GSH. This miniaturization also decreases solvent consumption, analysis time, and sample volume (Ishida et al., 2015). To the best of our knowledge, there is no report for application of portable HPLC in GSH determination. On the other hand, research on chromatography by microfluidics is progressing to allow separation with miniaturized columns through lab-on-a-chip technologies. Development is this area is highly expected to characterize changes in GSH levels in different pathological conditions. Microextraction of GSH samples could be a solution in traditional LC/UV methods for GSH determination in biological samples. Microextraction allows determination of low concentrations using small-volume organic solvents, which matches the current trends in green chemistry, miniaturization, and solvent preservation (Mansour \& Danielson 2017 ; 2018).

\subsection{Electrochemical methods for CSH analysis}

Glutathione is a key player in cellular regulation of oxidative stress because of its redox activity, which is the same reason why electroanalytical methods are suitably employed in its determination. Electrochemical methods are generally reliable, cost-effective, fast, and sensitive. They also offer the prospect of developing a sensor/strips suitable for use at point of care for diagnostic, monitoring, and follow-up purposes.

In normal situations, most of total GSH is present as GSH reduced form $(\sim 90 \%)$, which explains why the majority of methods are directed toward measuring the reduced form. The thiol proton is the most ionizable proton of GSH in basic media. The oxidation of GSH gives an electrochemical signal with a formal potential of $+0.23 \mathrm{~V}$ versus NHE. Upon oxidation, thiol compounds were reported to bind to the electrode surface (especially metals), which stops further detection. This problem was largely overcome in the reviewed methods by abandoning metal electrodes and investing in carbon-based electrodes with various modifiers. Cyclic voltammetry (CV) was the method of choice to characterize electrocatalytic properties of the proposed electrodes, whereas chronoamperometry was used to calculate diffusion coefficients and reaction rate constants. For quantitation, 
the produced current was measured by different methods (Table 2) which are more sensitive and accurate like differential pulse voltammetry (DPV), square wave voltammetry (SWV), and linear sweep voltammetry (LSV). 


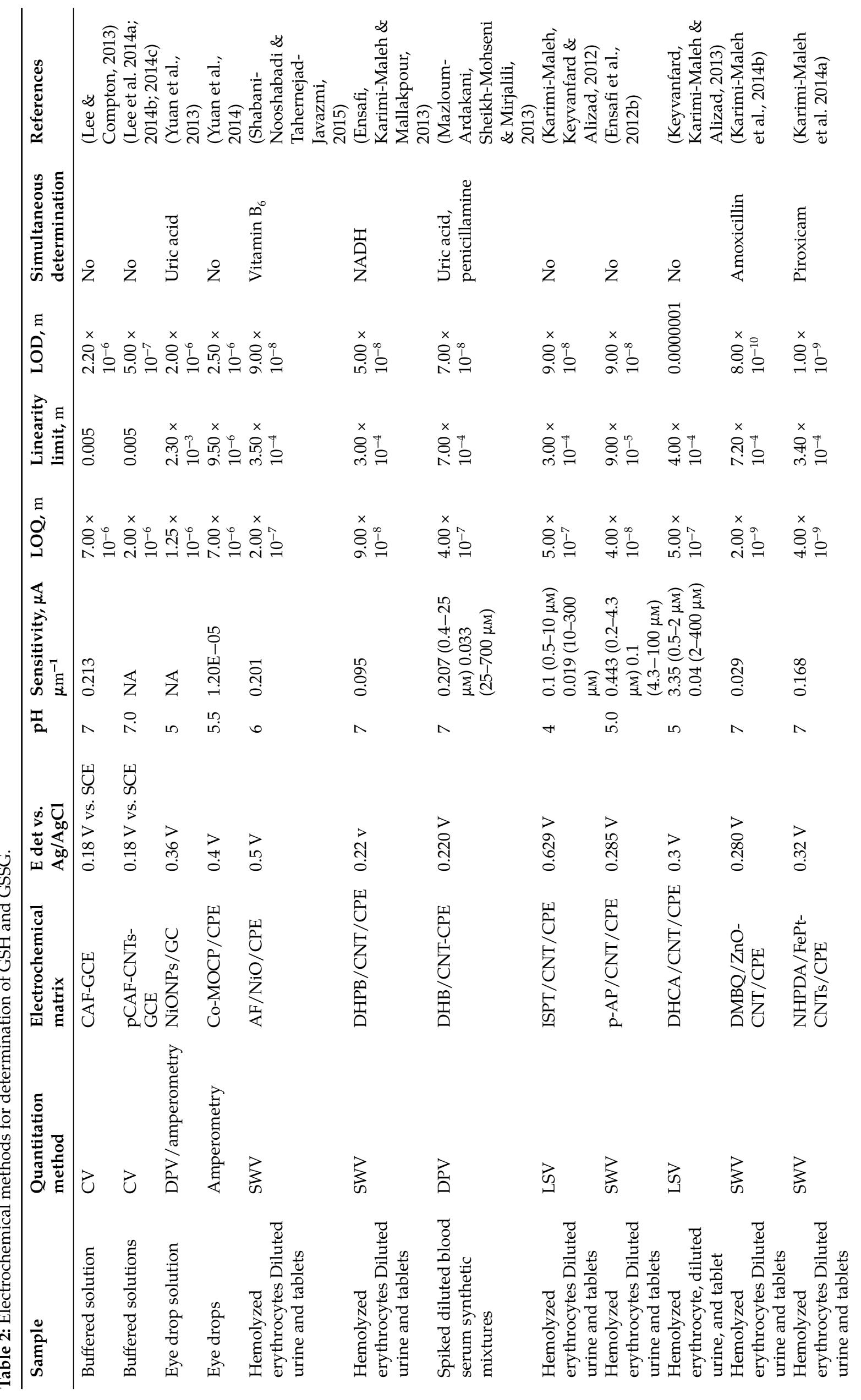




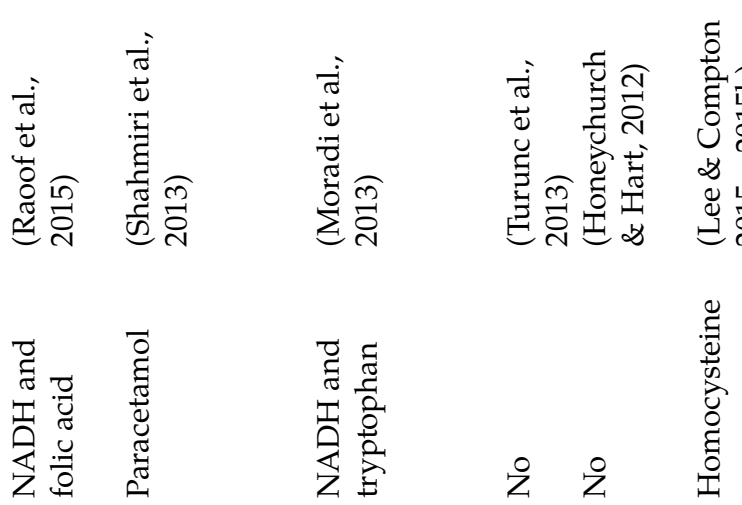

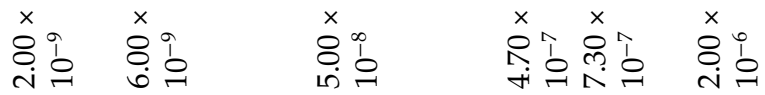

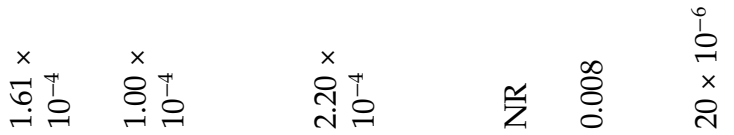

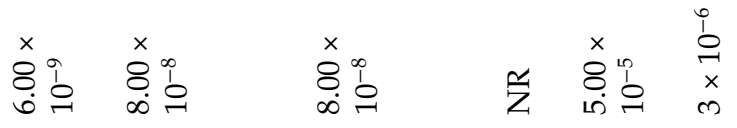

लै

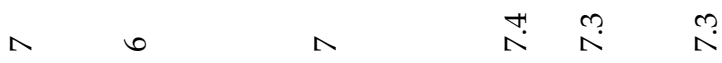

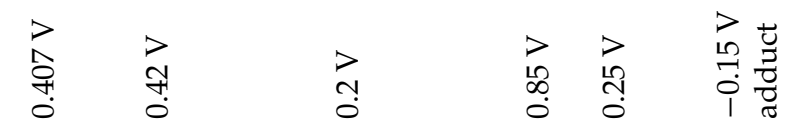

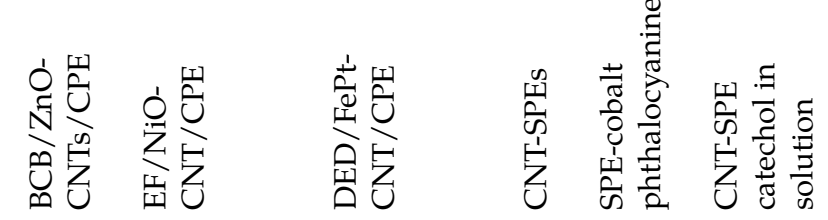

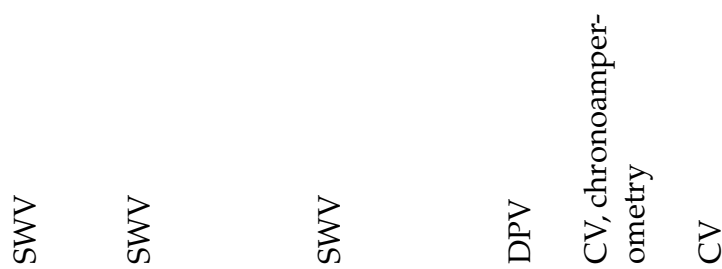

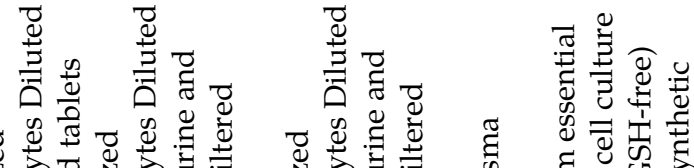

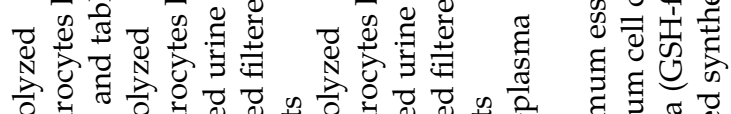

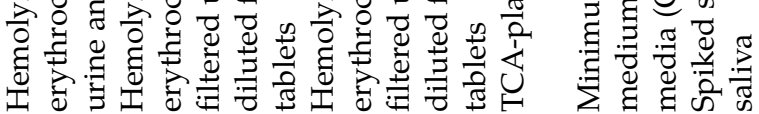


A good strategy was to try oxidizing GSH at lower positive potentials to avoid high background signals and interferences as well as electrode fouling. For this reason, modifications of GSH electrodes will be discussed in more details in the following section.

\subsubsection{Chemical modifiers in CSH electrodes}

As previously mentioned, redox mediators with larger diffusion coefficients and rapid reversible redox behavior are commonly employed in GSH electrodes to facilitate electron transfer between GSH and the electrode surface and to decrease electrode fouling. In addition, chemical modifiers increase the resultant current, which allowed for lower detection limits. However, leaching out of the chemical modifier from the electrode surface negatively affects the stability and reproducibility. This was less of a problem when using modifiers of lower water solubility.

\subsubsection{Selectivity and sensitivity of CSH electrodes}

Selectivity is defined as the ability of an analytical method to accurately and precisely determine the analyte of interest in the presence of other expected interfering substances such as ascorbates, Cys, and urate, which also have similar oxidation potential. Glutathione could be simultaneously determined with other compounds through using different redox mediator or through applying different scan rates to the same sample (Lee \& Compton 2015a; 2015b).

Glutathione concentration falls in the mм range inside mammalian cells, whereas in plasma, urine, and saliva, it is found to be in the $\mu \mathrm{m}$ range. That explains the necessity to develop methods that attain lower LOD, especially for biological samples such as urine and saliva. Also, this staggering difference in concentration levels explains the importance of having a wide dynamic range to cover such a large concentration difference. Having a wide dynamic range is one of the strengths of electrochemical methods, whereas the use of modifiers (chemicals and nanostructures) pushed the LOD lower and lower to the nм range (Table 2).

Sensitivity is important as well to accurately reflect small fluctuations in GSH levels that are clinically important. Some of the modified electrodes showed staggeringly different sensitivities over different concentration ranges (Table 2). This could be - to a certain extent - the result of adsorption of GSH on the electrode surface at higher concentrations or the limitations of diffusion.

\subsubsection{Types of electrodes used in GSH determination}

\subsubsection{Classy carbon electrodes}

The glassy carbon electrode is one of the most common working electrodes in GSH analysis due to its wide electrochemical window. Lee and modified the surface of a glassy carbon electrode (GCE) with electropolymerization of caffeic acid, which resulted in deposition of polycaffeic acid. This polymer facilitates the electron transfer between analytes (one of which was GSH) and the carbon electrode during the electrochemical oxidation process, due to the redox properties of the quinone moiety of the polycaffeic acid. This electrocatalytic activity was evident from the cyclic voltammogram, and the electrode was also used for determining nicotinamide adenine dinucleotide (NADH) and Cys separately. The method showed high sensitivity $\left(213 \mu \mathrm{A} \mathrm{mM}^{-1}\right)$, low LOD $(2.2 \mu \mathrm{M})$, and wide dynamic range (up to $5 \mathrm{~mm}$ ), all in buffered solution of GSH alone.

Improvements were introduced by Lee et al. (2014c) through investigating the effect of depositing carbon nanotubes (CNTs) by drop-casting onto the electrode surface before electrochemical polymerization and immobilization of polycaffeic acid film. Carbon nanotubes increased the polymer surface coverage by three-folds, which led to an increase in method sensitivity and reproducibility. The more economic nanocarbon clusters showed 12-fold increase in surface coverage, but this was accompanied by a decrease in sensitivity, probably because of the steric effect. The LOD was indeed lower than its predecessor (Lee \& Compton, 2013) $(0.5 \mu \mathrm{M})$ while maintaining its wide dynamic range.

The use of electrodeposited nanoparticles to modify GCE surface was reported by Yuan et al. (2013). They opted for $\mathrm{NiO}$, which markedly lowered oxidation potential for $\mathrm{GSH}(+0.36 \mathrm{~V})$ and was capable of quantifying it in eye drops preparation and simultaneously determined uric acid. The LOD was $2 \mu \mathrm{m}$, whereas the dynamic range was up to $2.3 \mathrm{~mm}$. In all three reports, GCEs were not applied to biological samples. Their behavior was rather explored in buffered solutions. 


\subsubsection{Carbon paste electrodes}

The majority of the reviewed methods utilized carbon paste electrode (CPE) with different modifications (Nesakumar, Berchmans \& Alwarappan, 2018; Panraksa et al., 2018). Carbon paste electrode is versatile and easy to construct, modify, and operate. All the reported methods showed very low detection levels that were enhanced by adding chemical modifiers and nanomaterials. Carbon nanotubes were the most popular addition to CPE; however, few other methods opted for different options (Yuan et al., 2014) (Shabani-Nooshabadi \& Tahernejad-Javazmi, 2015).

Yuan et al. (2014) chose adding a 2-dimensional cobalt-based metal-organic coordination polymer to the CPE. Their electrode showed high stability and was successfully applied for amperometric determination of GSH in eye drops with an LOD of $2.5 \mu \mathrm{m}$ and a wide dynamic range up to $0.95 \mathrm{~mm}$. However, the sensitivity was very low $\left(12.13 \mathrm{nA} \mathrm{m}^{-1}\right)$. It was suggested by the author - after running an interference study - that this electrode might be suited to intracellular measurement, where the high level of GSH (mm) would allow its determination in the presence of interfering substances after suitable dilution.

In another method (Shabani-Nooshabadi \& Tahernejad-Javazmi, 2015) used NiO nanoparticles instead of CNTs with acetylferrocene as a chemical redox mediator in CPE. Using SWV, the electrode showed a wide dynamic range $(0.2-350 \mu \mathrm{M})$, a low LOD $(0.09 \mu \mathrm{M})$, and a high sensitivity of $0.2011 \mu \mathrm{A} \mu \mathrm{M}^{-1}$. They were able to simultaneously determine GSH and vitamin $\mathrm{B}_{6}$ as their peaks were well separated with no compromise on. The electrode showed good recovery of GSH (comparable to standard method) in hemolyzed erythrocytes, spiked urine, and tablet solutions.

The electrochemical features of CNTs make them suitable for use in Faradaic processes. They have a higher electrical conductivity and can conduct high currents. They also offer a porous surface layer, which exhibits thin layer-like diffusion rather than semi-infinite diffusion described at metal electrode surface. This thin layerlike diffusion reduces the GSH transport rate to the extent that allows producing interpretable electrochemical signals. When comparing the performance of CPE with and without CNTs, it was clearly shown by CV that CNTs/CPE were superior in terms of observed current intensity and peak resolution. It also led in several reports to marked decrease of oxidation overpotential, which lowered electrode fouling (Ensafi et al., 2012a).

Carbon nanotubes were not used alone in most of the cases reviewed here; instead, redox mediators were added ( $1 \%-5 \%$ in most of them). These modifiers lead to a decrease in the potential required for GSH oxidation, as well as an increase in current intensity compared to CNT/CPE. Hence, with all reported modifiers, an increase in sensitivity and the decrease in LOD were observed when compared to CPE with only CNTs (Table 2). All of those electrodes were successfully used to assay GSH in hemolyzed erythrocytes, spiked diluted filtered urine, and diluted filtered tablet solution with an acceptable accuracy. Different electrochemical techniques were used including SWV (Ensafi, Dadkhah-Tehrani \& Karimi-Maleh, 2012b; Ensafi, Karimi-Maleh \& Mallakpour, 2013), LSV (Karimi-Maleh, Keyvanfard \& Alizad, 2012; Keyvanfard, Karimi-Maleh \& Alizad, 2013), and DPV (Mazloum-Ardakani, Sheikh-Mohseni \& Mirjalili, 2013).

The different chemical modifiers used in those studies gave comparable LOD and were mostly applied for assay of GSH alone with a few exceptions (Ensafi, Karimi-Maleh \& Mallakpour, 2013; Mazloum-Ardakani, Sheikh-Mohseni \& Mirjalili, 2013), where other analytes were determined simultaneously. Using N-(3,4-dihydroxyphenethyl)-3,5-dinitrobenzamide as a mediator (Ensafi, Karimi-Maleh \& Mallakpour, 2013) enabled simultaneous determination of GSH and NADH, whereas the use of (E)-3-((2-(2,4dinitrophenyl)hydrazono)methyl)benzene-1,2-diol enabled simultaneous determination of GSH, uric acid, and penicillamine in synthetic mixtures containing all three and spiked diluted blood serum sample using standard addition method (Mazloum-Ardakani, Sheikh-Mohseni \& Mirjalili, 2013).

The sensitivity for all of those mediators was different for different concentration ranges, being very high for lower-concentration segments and markedly decreased for higher concentrations. This could be because of saturation of electroactive sites. Analysts can choose the most appropriate modifier for their desired concentration range.

After investigating the positive effect of adding CNTs to chemical modifier (mediator)/CPE, the next step was to make nanocomposites electrodes. These new electrodes were prepared by treating the CNTs with strong acids before depositing various metals / oxides. The newly formed nanocomposites were carefully characterized using X-ray diffraction (XRD) and transmission electron microscopy (TEM) before adding them to a mixture of the chosen modifier and graphene.

Electrochemical characterization of modified nanocomposites electrodes clearly showed a marked increase in the catalytic activity evident by $\mathrm{CV}$, chronoamperometry, and electrochemical impedance spectroscopy studies. The use of such nanocomposite electrodes not only enhanced LOD to reach the nu level, but also seems to allow for simultaneous determination of GSH and other analytes of interest in hemolyzed erythrocytes, diluted filtered urine and diluted filtered tablet solutions. This could offer the possibility of measuring the levels of certain chemicals and examining their effect on the GSH level at the same time with minimal interference and high reliability. 
Amoxicillin was simultaneously analyzed with GSH using CPE modified by 8,9-dihydroxy-7-methyl-12Hbenzothiazolo[2,3-b]quinazolin-12-one (DMBQ) and ZnO/CNTs nanocomposite (Karimi-Maleh et al., 2014a, Karimi-Maleh et al., 2014b), which could be useful while studying amoxicillin toxicity as reflected by the GSH levels. Also, a CPE modified with N-(4-hydroxyphenyl)-3,5-dinitrobenzamide (NHPDA)/FePt-CNTs was able to successfully assay GSH and piroxicam simultaneously (Karimi-Maleh and Tahernejad-Javazmi, 2014), while modifying it with ethynylferrocene (EF) and NiO-CNT (Shahmiri et al., 2013) enabled quantifying both GSH and paracetamol in the same run. Simultaneous quantitation of GSH, NADH, and tryptophan was reported (Moradi et al., 2013) using 9,10-dihydro-9,10-ethanoanthracene-11,12-dicarboximido-4-ethylbenzene-1,2-diol (DED) as mediator of CPE containing FePt-CNT nanocomposite. This electrode also presented the largest decrease in GSH oxidation potential. In all the reviewed methods utilizing nanocomposites, SWV was the technique of choice for quantitation after careful electrochemical characterization with $\mathrm{CV}$ and chronoamperometry.

\subsubsection{Screen-printed electrodes}

The ultimate goal is to reach a sensor that selectively measures GSH in biological samples with acceptable accuracy, cost, and sensitivity. Direct oxidation of GSH required high overpotential because of its slow electron transfer process. Thus, modifications of such electrodes were needed to oxidize GSH at as low potential as possible to couple it with disposable screen-printed electrodes (SPEs), which offer the possibility of mass production at low cost. This could open the door for introducing GSH measuring strips point-of-care sensors. The problem remains, however, in the selectivity of such strips when used with real biological samples. The reviewed reports, all measured at considerably low positive potentials, showed low LODs in the micromolar range but were variable in sensitivity.

Honeychurch and Hart (2012) reported the use of microband SPE modified with cobalt phthalocyanine for determination of GSH at micromolar levels in minimum essential cell culture media. They proposed it as a lead for cell metabolism studies as it utilized less sample - because of its smaller size - without sacrificing dynamic range or LOD. Although it is an improvement over their previous "macro" electrode (Wring et al., 1990) as it showed much greater current density, still its sensitivity was low compared to other methods, and more work is still needed to enhance its selectivity and sensitivity.

Turunc et al. (2013) investigated the usability of disposable CNT-modified SPEs to measure levels of GSH in rat plasma by DPV. The plasma had to be deproteinized with TCA to remove interference and achieve satisfactory results that were in agreement with a reported spectrophotometric method.

Compton's group extended their work on modified GCE and their use in determining of GSH (Lee \& Compton, 2013; Lee et al., 2014c) into a proof of concept to the use of multiwalled CNT SPEs with added catechol to solution for determination of GSH and HCys in cell tissue media (Lee et al. 2014a; 2014b; 2014c), human plasma (Lee \& Compton 2015a; 2015b), and synthetic saliva (Lee \& Compton 2015a; 2015b). They exploited the formation of adduct based on 1,4-Michael addition with catechol, which gave a distinct adduct peak in CV that was different from the mere oxidation/reduction of catechol encountered with interfering thiols (Cys). They also used the difference in reaction rate between HCys and GSH in forming the aforementioned adduct. Fast scan rate quantified only HCys, whereas the slower ones determined both. This is still an early stage, but they demonstrated the possibility of using ready-made SPEs to the determination of GSH in spiked synthetic biological samples that contain commonly encountered interference and GSH to allow for determination of each in the presence of each other with acceptable recoveries.

\subsubsection{Electrochemical methods for CSH determination: what is next?}

Electrochemical sensing of GSH has been recently reviewed (Hanko et al., 2019). Although current LODs for most electrochemical methods are below physiological levels, there is still an urgent need for lower LODs to correctly quantify reduced levels of GSH associated with various pathologic processes, such as autism, which shows very low levels of GSH in urine (Kern et al., 2011). The enhanced LOD would also permit more sample dilution, to guard against electrode fouling encountered with complicated biological samples. Higher sensitivity is also sought after to better study small fluctuations associated with some pathologic conditions. Researchers used to focus on buffered synthetic solutions rather than real physiological samples (Harfield, Batchelor-McAuley \& Compton, 2012). This trend has now dropped in favor of studying more realistic samples, but the challenge remains to achieve more selectivity to be suited for the complex nature of biological samples. Still more work is needed to reach a methodology/apparatus suitable for point-of-care testing. 


\subsection{Capillary electrophoresis in CSH determination}

Capillary electrophoresis (CE) is a separation technique that depends on the influence of an electric field that drives analytes toward the detector. Because separation is attained in narrow-internal-diameter capillaries, resolution is exceedingly good at the short assay time. Capillary electrophoresis is greener and more eco-friendly as it requires a minute sample, buffer, and solvents and generates minimal waste. Although many types of capillaries are available, all reviewed methods used uncoated fused silica capillaries of 25- to $75-\mu \mathrm{m}$ internal diameter, with the majority using $75 \mu \mathrm{m}$ (Table 3). This is mainly because of the short life of modified capillaries and the more care and maintenance they require.

Capillary zone electrophoresis was used by various researchers (Kubalczyk \& Bald, 2009; Li, Cheng \& Tseng, 2009; Mendoza et al., 2009; Yan et al., 2009; Chang \& Tseng, 2010; Shen, Tseng \& Hsieh, 2012; Tsardaka et al., 2013; Wang et al., 2014a; Wang et al., 2014b), but it does not appear to offer a specific advantage in terms of sensitivity when used alone without derivatization (Kubalczyk \& Bald, 2009; Mendoza et al., 2009; Yan et al., 2009; Tsardaka et al., 2013; Wang et al., 2014a; Wang et al., 2014b). However, it surely allowed shorter analysis times. Because it allows preconcentration of the sample, there is less need for derivatization, which makes it simpler, faster, and more economic than other techniques.

Different strategies were reported. Some opted for modifiers that suppressed electro-osmotic flow such as poly(diallyldimethylammonium chloride) (PDDAC). Poly(diallyldimethylammonium chloride allowed very sensitive detection even with direct UV detection (Li, Cheng \& Tseng, 2009; Chang \& Tseng, 2010). They greatly enhanced the sensitivity to reach an unprecedented LOD $\left(1 \times 10^{-11} \mathrm{M}\right)$ by the addition of polymers to the buffer. This strategy will be discussed in more details in Section 2.3.6 (Shen, Tseng \& Hsieh, 2012).

On-capillary acetonitrile stacking was the chosen strategy in one report (Kubalczyk \& Bald, 2009), which works through transient pseudo-isotachophoresis and allows for larger sample volumes to be injected. Acetonitrile (ACN) supplied high field strength needed to speed up the analytes' migration; thus, it works as a pseudo-terminating ion.

Transient isotachophoresis - which conveniently does not require changes in the capillaries - was the strategy chosen for assay of GSH and GSSG in different samples (Yan et al., 2009). First, almost three-quarters of the capillary - which was initially full of leading electrolyte - was filled with the sample "plug." Next, a "plug" of the terminating electrolyte was injected to guard against sample escape. Negative voltage was then applied to concentrate analytes (analytes moving against the electro-osmotic flow toward inlet). Finally, separation was initiated through using positive potential $(25 \mathrm{kV})$.

Online stacking was the reason for the high sensitivity of UV detection methods (Li, Cheng \& Tseng, 2009; Chang \& Tseng, 2010). Acetate buffer at low $\mathrm{pH}$ was used - imparting positive charge to analytes - and the capillary was incubated overnight with PDDAC reagent. Separation was performed while the capillary was still filled with the reagent to allow for sample concentration at the interface between sample and PDDAC zones where the positively charged analyte ions slow down - because of change in viscosity and decay of electric field - until reversed electro-osmotic flow carries them to the detector. This meant that the separations were conducted at negative potential (unlike all other reviewed work, which operated at various positive potentials). This method (Chang \& Tseng, 2010) was proposed for the determination of total aminothiols (reduced, oxidized, and protein bound through a first step of reduction with tris(2-carboxyethyl)phosphine), whereas its predecessor (Li, Cheng \& Tseng, 2009) quantified free GSH, Cys, and HCys in spiked human urine without the reduction step.

Much lower LOD (0.1 pM) was reported by the same group when derivatization followed by online stacking was employed (Shen, Tseng \& Hsieh, 2012). This was achieved through using electro-osmotic flow that was greater than the effective electrophoretic mobility of OPA-derivatized peptides to make these negatively charged derivatives enter the neutral poly(ethylene oxide) zone, thus enhancing their separation. Poly(ethylene oxide) gets adsorbed on the capillary leading to decrease in electro-osmotic flow mobility, which prolongs the migration time, thus separating closely adjacent peaks. It is to be noted that analysis time approached $8 \mathrm{~h}$ (Shen, Tseng \& Hsieh, 2012). 


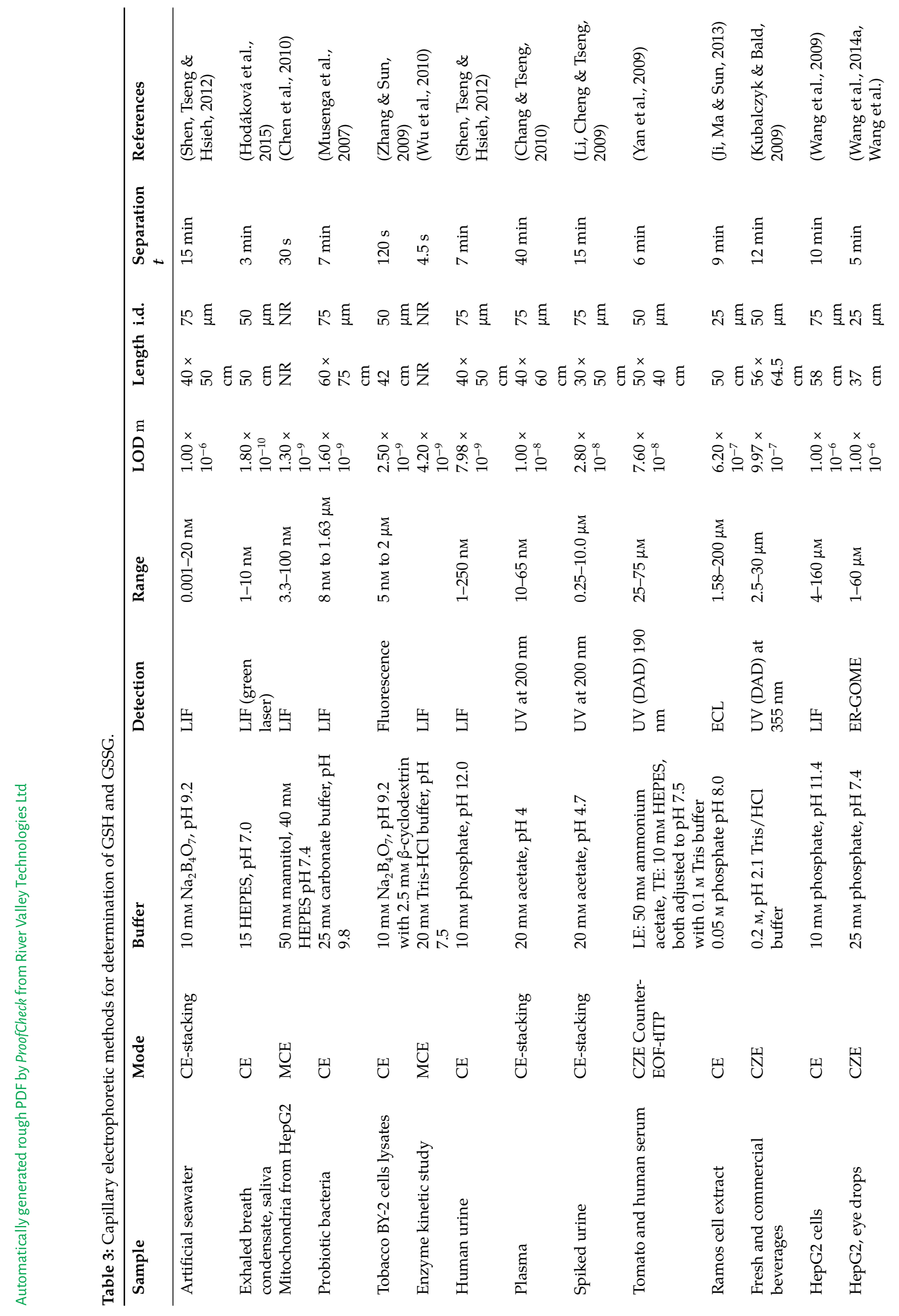




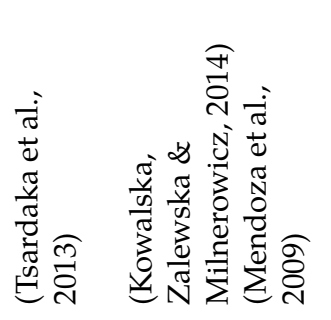

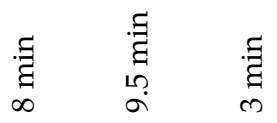

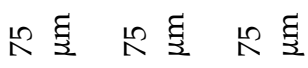

늄

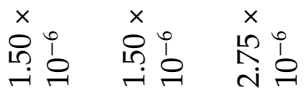

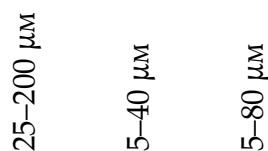

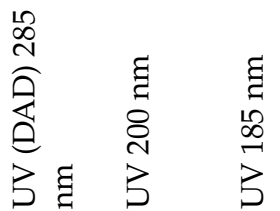

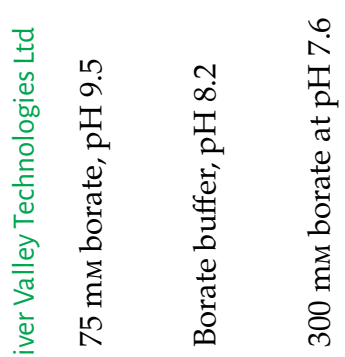

ูํำ ํㅓㄴ 


\subsubsection{Sample types and preparation in CE}

The reported CE methods were applied to biological samples with great diversity ranging from plants (Kubalczyk \& Bald, 2009; Mendoza et al., 2009; Zhang \& Sun, 2009), probiotic bacteria (Musenga et al., 2007), yeast (Tsardaka et al., 2013), and animals (Wang et al., 2009; Chang \& Tseng, 2010; Chen et al., 2010; Wu et al., 2010; Shen, Tseng \& Hsieh, 2012; Ji, Ma \& Sun, 2013; Hodáková et al., 2015), and in one report, both animal and plant applications on tomato and human serum (Yan et al., 2009). Two methods reported success in assaying GSH in individual cells with high sensitivity (Ji, Ma \& Sun, 2013; Wang et al., 2014a; Wang et al., 2014b). Pretreatment of samples usually involved dilution and filtration. Sometimes (Musenga et al., 2007), protein precipitation was required; in such case, the use of organic solvent was preferred to avoid oxidation caused by acids. The use of acids would also have incurred an increase in salt concentration when trying to neutralize them for derivatization and/or separation. This increase in salt concentration is not advised in CE.

\subsubsection{Simultaneous determination}

Its power as separation techniques was demonstrated by its ability to simultaneously determine other analytes of interest to give a better understanding of oxidative stress.

Hydrogen peroxide was assayed together with GSH in mitochondria of apoptotic cells (Chen et al., 2010); GSSG was determined in the same run (Mendoza et al., 2009; Yan et al., 2009; Kowalska, Zalewska \& Milnerowicz, 2014) to investigate if a decrease in GSH is exclusively translated into an increase in GSSG. Another study was interested in measuring both free and protein-bound GSH (Wang et al., 2009) where tri-n-butylphosphine was used to cleave the disulfide bond to release it and then proceeded as in free GSH. This helped better understand protein S-glutathionylation in intact cells, which generally occurs in response to mild oxidative stress and is implicated in cell signaling. The authors proposed that their method could be adapted to study the effect of various chemicals on protein S-glutathionylation. Phytochelatins (Shen, Tseng \& Hsieh, 2012), HCys, and $\gamma$-glutamylcysteine (Shen, Tseng \& Hsieh, 2009) were derivatized and quantified together with GSH using o-phthaldehyde. Four other aminothiols, -glutamylcysteine, Cys, HCys, and cysteineglycine, were selectively extracted, separated. and quantified with GSH in plasma (Chang \& Tseng, 2010). Cysteine and HCys were directly determined with GSH in spiked urine samples (Li, Cheng \& Tseng, 2009), whereas Cys alone was well resolved in different beverages (Kubalczyk \& Bald, 2009).

\subsubsection{Speed of analysis}

The fastest two methods by far required only few seconds for separation and minimal time for derivatization. The first (Wu et al., 2010) developed microchip CE with on-column derivatization and required only $4.5 \mathrm{~s}$ for successful separation. The second method (Zhang \& Sun, 2009) achieved successful derivatization in 4 min, whereas separation took only $2 \mathrm{~min}$. Direct determination with no derivatization was also fast ( $<10 \mathrm{~min})$; here, the complexity of samples played a critical role (Table 3).

Separation time was generally less than $15 \mathrm{~min}$ in the reviewed work, with the majority even less than 10 min (Table 3). Only one method required exceedingly long separation time of 40 min (Chang \& Tseng, 2010). Most of the analysis time was spent equilibrating capillary and processing the sample for enrichment and derivatization. Some methods required exceedingly long reaction times. An overnight incubation in the fridge was required in one of them to avoid degradation of the reagent (Hodáková et al., 2015), whereas others using Tween 20-capped gold NPs (AuNPs) for extraction/sample enrichment of GSH required prolonged periods for extraction and subsequent release of the analyte (Li, Cheng \& Tseng, 2009; Shen, Tseng \& Hsieh, 2009; Chang \& Tseng, 2010; Shen, Tseng \& Hsieh, 2012).

\subsubsection{Detection}

The lack of a good chromophore in GSH meant that direct UV detection is possible only at 180-200 nm, which, although direct and fast, takes its toll on selectivity and increased background peak/noise (Li, Cheng \& Tseng, 2009; Mendoza et al., 2009; Chang \& Tseng, 2010; Kowalska, Zalewska \& Milnerowicz, 2014). Another approach was to exploit the redox characters of GSH through electrogenerated chemiluminescence (ECL) using tris(2,20bipyridine) ruthenium(II) (Ji, Ma \& Sun, 2013) or direct measurement with a modified carbon electrode (Wang et al., 2014a, Wang et al.). 
The most popular approach was to derivatize the analytes of interest into chromophores (Kubalczyk \& Bald, 2009; Tsardaka et al., 2013) or fluorophores for even lower LOD. Most reviewed articles used fluorometric detection (Musenga et al., 2007; Shen, Tseng \& Hsieh, 2009; Wang et al., 2009; Chen et al., 2010; Wu et al., 2010; Shen, Tseng \& Hsieh, 2012; Hodáková et al., 2015) and nonlaser fluorometry (Zhang \& Sun, 2009).

\subsubsection{Derivatization}

The reviewed methods used a variety of derivatizing reagents mainly to enhance detectability. 5Iodoacetamidofluorescein - a common fluorogen - was used by two groups (Musenga et al., 2007; Wang et al., 2009). When used with prokaryotes cells (Musenga et al., 2007), they chose to derivatize at $40{ }^{\circ} \mathrm{C}$ as it decreased the required reaction time. But when applied to more complex hepatoma cells (Wang et al., 2009), the higher temperature led to increased background peaks, which made the researchers switch for $90 \mathrm{~min}$ at room temperature.

Two studies (Shen, Tseng \& Hsieh 2009; 2012) used another fluorogen, OPA, which reacts with the amino group in the presence of the nucleophilic sulfhydryl group without the addition of another nucleophile. This reagent was advantageous because it does not react with a major interference, Cys, in the absence of external nucleophile.

A similar fluorescence derivatizing reagent, naphthalene-2,3-dicarboxaldehyde (NDA), was also employed (Zhang \& Sun, 2009). This reagent was similar in its reaction mechanism to OPA, but it had a major advantage - the reaction time was only $4 \mathrm{~min}$ at room temperature. The separation time of 2 min meant this method applied in tobacco BY-2 cells lysates, the HeLa cells of higher plants - was one of the fastest reviewed methods. $\beta$-Cyclodextrin was added to the running buffer to enhance sensitivity and migration speed.

Rh-Se-2, a nonfluorescent rhodamine-based organoselenium fluorescent probe, was used for determination of GSH in mitochondria of apoptotic cells (Chen et al., 2010). This reagent reacted with GSH in fairly mild conditions $\left(37^{\circ} \mathrm{C}\right.$ for $30 \mathrm{~min}$ ) to produce the highly fluorescent rhodamine 110 with high quantum yield.

An even faster reagent in reacting with GSH to produce a fluorescent product was thioglo- $1, \mathrm{~N}-(2-$ carbomethoxy-9-methoxy-3-oxo-3H-naphtho[2,1-b]pyran-10-yl)maleimide) (Wu et al., 2010). Although this reagent was previously used offline with 20-min reaction time, they demonstrated here that the reaction kinetics allow it to be used online with acceptable reproducibility, accuracy, and precision.

Because of the current wide availability of semiconductor laser modules with different excitation wavelength, eosin-5-maleimide was used to label GSH for detection by green laser $(515 \mathrm{~nm})$ and enabled its detection at less than $1 \mathrm{~nm}$ in saliva and exhaled breath condensate (Hodáková et al., 2015). Excitation at a longer wavelength is preferable for biological samples as it minimizes background/autofluorescence of matrix and offers more flexibility and selectivity for derivatization because they enable usage of derivatizing reagents with excitation wavelength outside that dictated by commercial laser-induced fluorescence (LIF) detectors. These modules are robust, economic, and small and operate at a low voltage. Although this method gave exceedingly low LOD, it required long derivatization reaction (at least $3 \mathrm{~h}$ and preferably overnight in the fridge).

To better detect GSH in different fresh and commercial beverages, a UV-derivatizing reagent was used (Kubalczyk \& Bald, 2009). 2-Chloro-1-methylquinolinium tetrafluroborate took only $10 \mathrm{~min}$ to form quinolinium derivatives detected at $355 \mathrm{~nm}$, hence offering more selectivity and less background noise while adequately resolving Cys and GSH. ACN stacking was used to enhance sensitivity of this method because it allowed injecting bigger sample volumes.

A group of researchers (Tsardaka et al., 2013) noted the problems encountered in derivatization revolved around long reaction times, cost, or restricted availability of reagents. They introduced alkyl propiolate as a commercially available, economic UV-labeling agent that offers fast derivatization at room temperature. The method was successfully applied to assay of GSH in Baker's yeast samples.

\subsubsection{Use of nanoparticles}

Four of the reviewed articles used Tween 20-capped AuNPs (Tween 20-AuNPs) for the selective extraction/enrichment of various aminothiols (Li, Cheng \& Tseng, 2009; Shen, Tseng \& Hsieh, 2009; Chang \& Tseng, 2010; Shen, Tseng \& Hsieh, 2012). Samples were incubated with Tween 20-AuNPs, followed by separation and washing of the analyte-adsorbed NPs and then release of analytes via dithiothreitol (Chang \& Tseng, 2010; Shen, Tseng \& Hsieh, 2012) or thioglycolic acid (Shen, Tseng \& Hsieh, 2009). Tween 20 capping was introduced both to help better disperse AuNPs in the sample and to inhibit the nonspecific adsorption; thus, it could be successfully applied for extraction of different biological samples. Fluorescence derivatization was tried (Shen, Tseng \& Hsieh 2009; 2012) using OPA (which meant Cys - a major interferent - could not react). But this added step 
required at least $2 \mathrm{~h}$ more and did not seem to add tangible advantage in biological sample when compared to the methods with direct UV detection without any derivatization (Li, Cheng \& Tseng, 2009; Chang \& Tseng, 2010). Although separation time was much longer in the UV detection method when applied to plasma (40 min but could be reduced to $25 \mathrm{~min}$ on shorter capillary), still overall analysis time was at least $2 \mathrm{~h}$ shorter and much simpler. Moreover, the UV method (Chang \& Tseng, 2010) was applied on plasma where Cys was well resolved and had a comparable LOD to its LIF counterpart used in "spiked" urine samples (Shen, Tseng \& Hsieh, 2009).

\subsubsection{Use of microchip capillary electrophoresis}

There were two reports of the development of microchip capillary electrophoresis (MCE) with excellent detection in the nм range. The first report developed an integrated microfluidic chip for on-capillary derivatization of GSH using the commercial reagent ThioGlo-1 (Wu et al., 2010). The produced fluorophore was detected by LIF and was useful for enzyme kinetic study. The developed system had the advantage of continuous monitoring capability of GSH. This was done using computer-controlled flow-gated injection with riboflavin as an internal standard to guard against any variability in injection volume.

The second report used two fluorescent probes to allow for simultaneous determination of GSH and hydrogen peroxide. Rh-Se-2, a rhodamine-based organoselenium for GSH and bis(p-methylbenzenesulfonate) dichlorofluorescein for $\mathrm{H}_{2} \mathrm{O}_{2}$. This method allowed separation in $30 \mathrm{~s}$, but needed $30 \mathrm{~min}$ for the derivatization reaction (Chen et al., 2010).

\subsubsection{Pros and cons}

The absence of stationary phase - with which some matrix constituents could interact irreversibly, hence fouling the column - meant less sample pretreatment is required in CE compared to chromatographic techniques. Simpler sample pretreatment in CE - only filtration and dilution in most cases - not only saves time, but also minimizes changes in GSH levels related to pretreatment steps. Such changes most importantly included oxidation of GSH, which lowered its measured level and hemolysis, which increased GSH levels in plasma. Other advantages included the simplicity of the procedure, minimal injection volume, and possibility of automation.

Migration times were very reproducible in all reviewed methods (\%RSD (relative standard deviation) $<2 \%$ ), whereas those of the area under the peak were variable, ranging from $1 \%$ to $5 \%$. Standard addition (spiking) and internal standards were commonly used to compensate for matrix effect and attain higher accuracy and precision. Internal standards included NAC (Tsardaka et al., 2013; Wang et al., 2009), L-penicillamine (Musenga et al., 2007), and proflavine, which was chosen in one of the MCE methods used for the enzymatic study of GSH reductase through following GSH and NADPH (Wu et al., 2010).

As discussed above, in many cases to enhance sensitivity and attain lower LOD, analysis time was prolonged by added steps of derivatization and enrichment/extraction. Also, many injection parameters (volume, pressure, time, and voltage) needed to be optimized. Careful control of $\mathrm{pH}$ was also essential and more critical compared to chromatography because in CE separation is dependent on charge/ionization, which is directly affected by $\mathrm{pH}$ (especially when CZE was employed).

One of the major limitations of CE that restricts its use is having an LOD inferior to modern chromatographic techniques. This is because of its very design (the use of capillaries), which, although it offers great advantages (speed, resolution and economy), it - unfortunately - imparts a short path length, and only minute sample volumes can be injected. This limitation was addressed through derivatization (Kuśmierek et al., 2011) to enhance the detectability (also protects the thiol group from oxidation) and using more sensitive detectors such as fluorimetry especially those using laser for excitation.

\subsection{Spectroscopic probes and sensors}

The field of fluorescence has witnessed a rapid-pace advance in the last two decades, during which, many novel classes of probes have been developed like semiconductor nanoparticles or quantum dots (QDs) and the genetically engineered green fluorescent probes. Also, a large number of new derivatives of known dye classes (like dansyl, fluorescein, rhodamine, and cyanine) were designed to improve certain aspects of the assay or add a new functionality, for example, improving specificity for a certain analyte or adding moieties that enhance targeting a specific cellular component. Another example of the new fluorescence probes is the molecular devices that utilize known fluorescent dyes in a supramolecular structure and molecular switches such as molecular beacons. The field also witnesses the increasing ability to develop fluorescent dyes with high degree of con- 
trol over the excited state and engineering them to exhibit particular sensitivity toward certain environmental conditions. The example for that is the new luminescent transition metal-ligand complexes (MLCs).

Surface-enhanced Raman spectroscopy (SERS) (Sánchez-Illana et al., 2018; Yao et al., 2019) is also gaining a lot of attention in recent years because of its great potential in detection of very small concentrations of analytes (down to a single molecule detection). The detection and quantitative determination of GSH have been one of the direct applications of many of the aforementioned new approaches in fluorescence spectroscopy and SERS. The chemical derivatization reactions used for spectroscopic detection of GSH are shown in Figure 2. Examples from the literature of these novel methods shall be discussed in the following sections and are summarized in Table 4

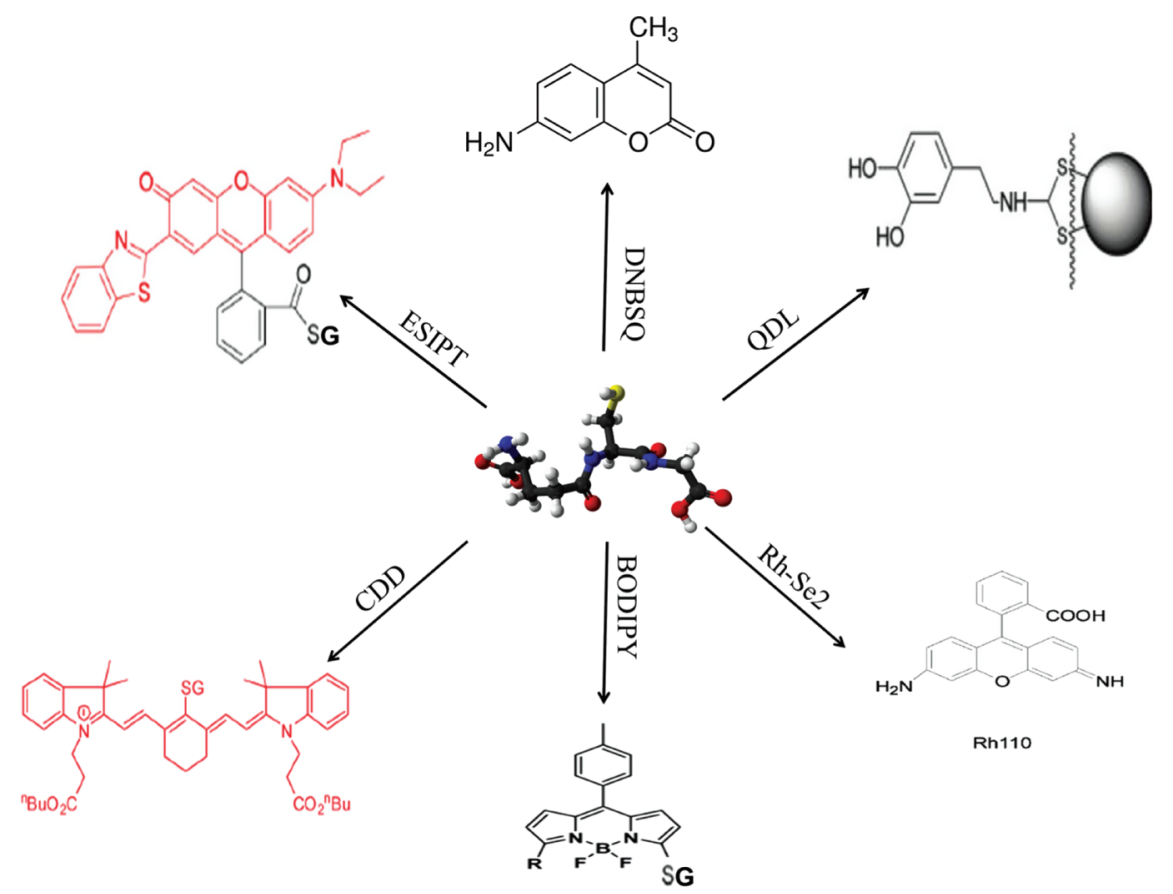

Figure 2: Chemical derivatization reactions used for spectroscopic detection of GSH.

\subsubsection{Nanomaterials and nanoclusters}

The realm of nanotechnology is expanding continuously to influence almost every applied field, and analytical chemistry is not an exception. Many nanoparticles are now commercially available and used in different analytical methods and techniques to improve sensitivity and specificity.

Quantum dots are considered a novel and extremely interesting type of nanomaterials. They have the ability to absorb and emit light. This is attributed to the difference in the semiconductor band gap between the core and the shell, which results in absorption of photons and creation of an electron-hole pair. Upon the recombination of this pair, the emission of a photon can occur.

One of the strategies for fluorescence detection is fluorescence light switch probes. In those probes, the presence of a specific analyte triggers a physical or chemical change in the molecular device that switches the fluorescence ON or OFF. Banerjee et al. (Banerjee et al., 2009) utilized the reducing ability of GSH to cleave the disulfide bond that tethers a quencher to the fluorescent QDs, thus turning it ON. This approach, however, is not specific to GSH, and other reducing substances in the sample could also break the disulfide bond.

The used fluorescent probe was a typical QD composed of CdS:Mn/ZnS core/shell nanoparticles with an average size of $4 \mathrm{~nm}$. The quencher used in this study was dopamine, which is an electron-rich molecule immobilized on the particle surface. Being in close proximity to the QD particles guarantees an effective electron transfer process. That makes it able to supply electrons to the particle. The resulting fast electron transfer disrupts the formation of electron-hole pair and consequently prevents emission of photons. The size of QDs is one factor in determining the fluorescence properties of the particles. The excitation wavelength of these particles is at $350 \mathrm{~nm}$, and emission was at $592 \mathrm{~nm}$. 


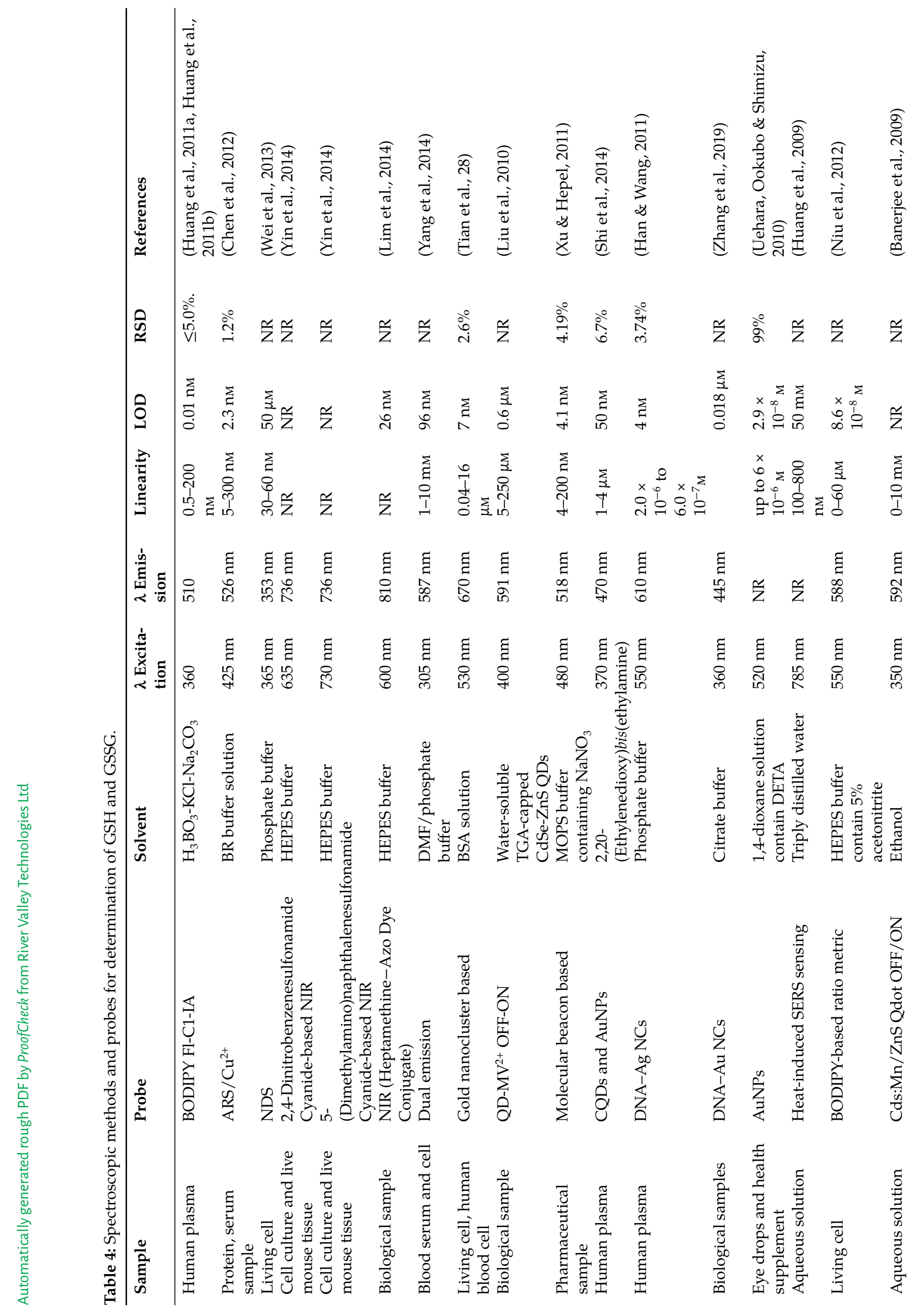




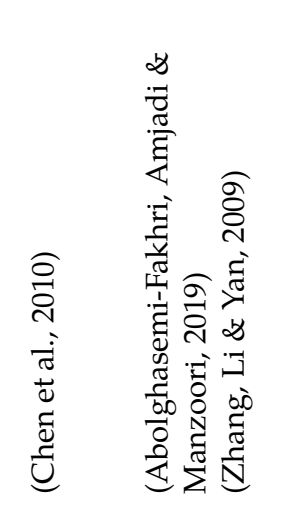

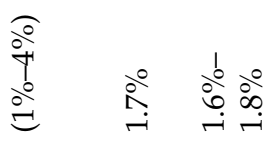

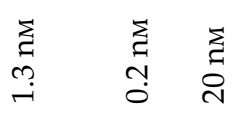

하요

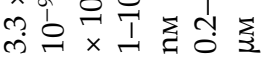

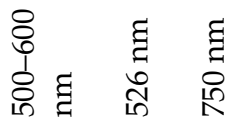

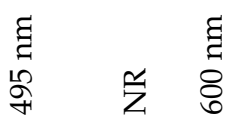

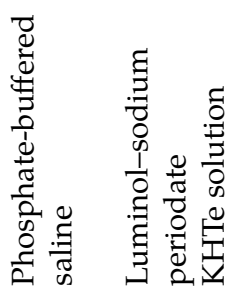


The QDs can be engineered to emit in other wavelengths. For example, GSH detection reported by (Zhang, Li \& Yan, 2009) used QDs that emit in the near infrared (NIR). The band gap for the core and shell for those QDs were tuned to have an emission maximum at $750 \mathrm{~nm}$. The QDs have CdTe/CdSe Core/shell structure with particle size of $5 \mathrm{~nm}$. Those particles were photo activated and then allowed to react with GSH-containing samples. The thiol group of the GSH binds to the particle surface and increases the fluorescence intensity of these particles via their electron donation ability. The thiol group acts as a scapegoat supplying electrons to the electron traps (quenchers), so it reduces the nonradiation decay of the QDs and hence increases the fluorescence. Like the previous example, this strategy lacks selectivity to GSH, as any thiolated compound can have the same effect, for example, Cys and HCys amino acids. However, it is unlikely that there is a fluorogenic compound in the sample that can emit at the NIR region, which means that the background fluorescence will be minimal and the sensitivity is improved.

To increase the specificity of the fluorescent QDs to GSH and reduce the interference from other chemically related compounds, Liu et al. (2010) utilized one structural property of GSH, which is the ability to form coordination complex with polyvalent metal ions as a basis for detection. This chelation power of GSH is much stronger than other thiolated amino acids such as Cys and Hcy, which could impart higher specificity to GSH. They used normal CdSe/ZnS core/shell QDs and decorated the surface with thioacetic acid (TAA), which forms an ion pair with the quencher methyl viologen (1,10-dimethyl-4,40- bipyridinium chloride, abbreviated as $\mathrm{MV}^{2+}$ ). Due to the ability of GSH to form a stable chelate with the metal surface, it can displace the TAA, which has the quencher attached to it (Figure 3A). This switches the particle to the ON state as the quencher is removed with the TAA. They proved that Cys and Hcy produce less fluorescence than GSH.

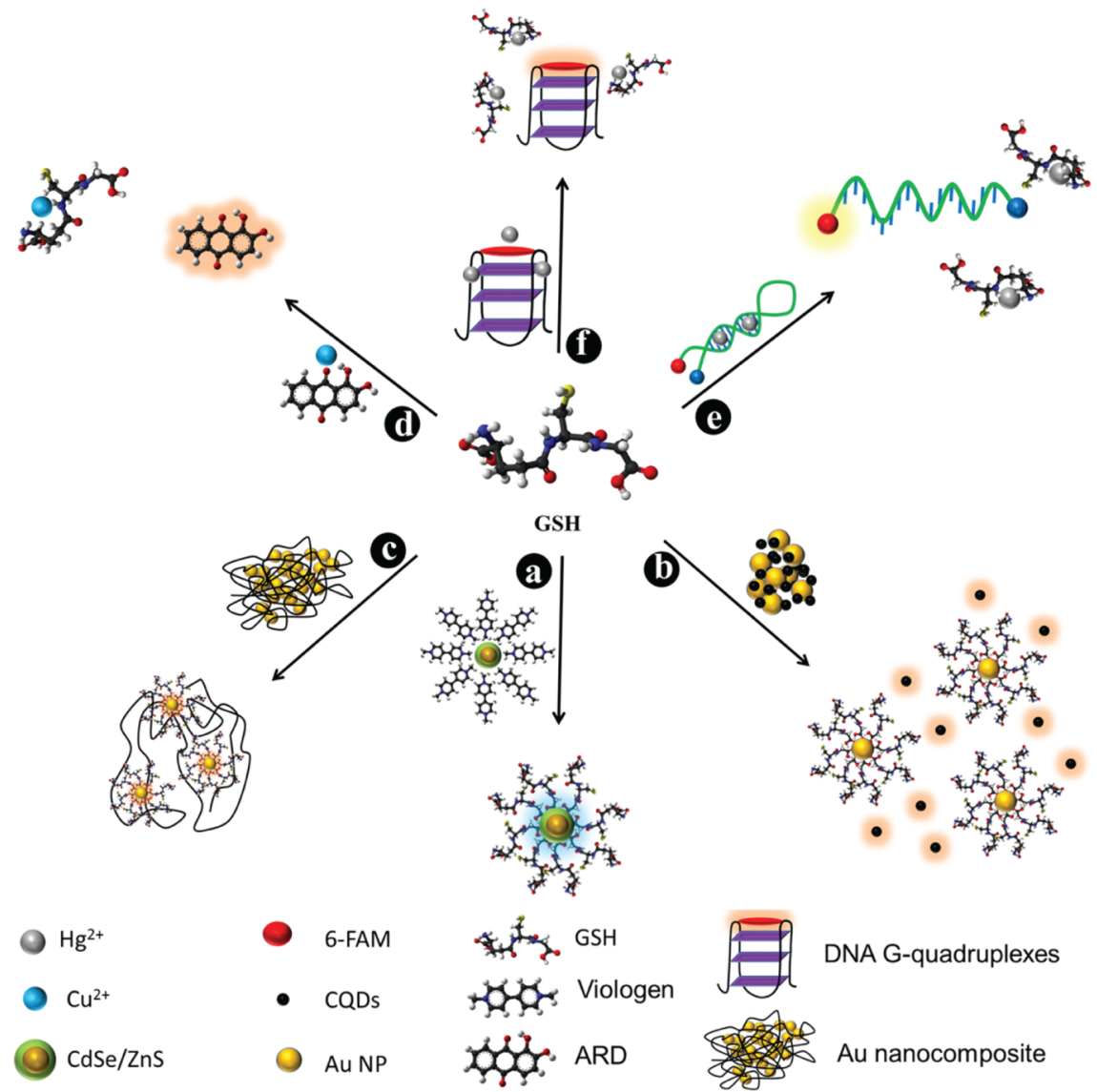

Figure 3: Principles of sensors used for determination of GSH.

Coinage metal nanoparticles, especially AuNPs are the most widely used nanomaterial in the field of nanotechnology. They have interesting photonic, catalytic, and spectroscopic properties. One of the interesting properties is that the light absorption wavelength is dependent on the size of particles and the spatial arrangement of particles in clusters (Nakamura \& Hayashi, 2005). Gold nanoclusters are known to have fluorescence properties. The spectroscopic characterization shows a very large stoke's shift with emission in the NIR region, which offers minimal self-quenching and lower background. Tian et al. (Tian et al., 28) prepared AuNCs by reducing Au solution in the presence of bovine serum albumin (BSA). This produced monodispersed AuNCs of average particle size $2.5 \mathrm{~nm}$. They showed stoke shift of almost $120 \mathrm{~nm}$ and emission at about $670 \mathrm{~nm}$. On the $\mathrm{Au}$ particles surface, a donor-acceptor bond is formed between the $\mathrm{Au}^{+}$and $\mathrm{Hg}^{2+}$ atoms. This binding causes 
quenching of the fluorescence. Glutathione binding to $\mathrm{Hg}^{2+}$ is much more favorable thermodynamically, so it strips $\mathrm{Hg}$ atoms from the particle surface, thus activating emission. This chelation with a divalent cation is happening only in case of GSH and to a much less extent with other biothiols, which makes this method highly selective to GSH.

Han and Wang (2011) used a recently introduced nanomaterial, namely, silver nanoclusters (Ag NCs), to detect biological thiols. One advantage of this material over other nanoparticles is being relatively less toxic and having better biocompatibility. The biothiols form coordination complexes with DNA-Ag NCs, which directly quenches Ag NCs emission. This is called the static quenching, which depends on the formation of a nonfluorescent ground-state complex between the fluorophore and quencher, which absorbs light and then immediately returns to the ground state without emitting a photon. This should be differentiated from the dynamic quenching at which the energy is transferred from an excited stated of the fluorophore to the quencher through the Förster resonance energy transfer (FRET). This conclusion about the static quenching was confirmed by measuring the photoluminescence decay lifetime of DNA-Ag NCs, which showed no significant change in case of the free DNA-Ag NCs and the ones complexed with biothiols. The fluorophore showed a large stokes shift (excitation wavelength at $550 \mathrm{~nm}$ and emission at $610 \mathrm{~nm}$ ) and a high quantum yield that takes the limit of detection down to 4 nm.

Shi et al. (2014) combined two different nanomaterials to create a molecular device that has two modes of measurement of GSH. They used colloidal mixture that consists AuNPs with a diameter of about $13 \mathrm{~nm}$ and fluorescent carbon QDs (CQDs). Carbon QDs function as fluorescent probe, and AuNPs have two roles: first to function as calorimetric reporter and second as a fluorescence quencher for the CQDs. The mechanism of the nanosensor is depicted in Figure 3B. Two distance-dependent phenomena occur simultaneously upon adding the analyte, color change of AuNPs suspension, and FRET. The addition of CQDs into AuNPs colloid solution may induce the aggregation of AuNPs and CQDs, leading to color change from red to blue and CQD fluorescence quenching. Glutathione has such high affinity to the Au surface that - when added - it breaks down the AuNPs aggregates. This enlarges the interparticle distance and subsequently produces a color change (back to red) and enhanced fluorescence intensity.

Uehara, Ookubo, and Shimizu (2010) used a water-soluble polymer to create Au nanocomposites from 13nm diameter $\mathrm{Au}$ nanoparticles. The color of the Au particles colloid changes from red to violet. The extinction spectra shifted toward a shorter wavelength from 680-nm plasmon extinction of the assembled AuNPs in a nanocomposite to $520 \mathrm{~nm}$, which is specific to separate AuNPs. The polymer is isopropyl acrylamide copolymer, and it tethers the particles together via amine groups that forms N-Au bond. Upon the introduction of GSH, it competes with the polymer at the Au surface and displaces the nitrogen atoms to form the more thermodynamically favorable sulfur-Au bond. This displacement of the polymer away from the Au particle surface disintegrates the nanocomposite into separate AuNPs. This changes the color back to red (colorimetric determination). The mechanism of detection is illustrated in Figure 3C.

\subsubsection{Molecular ensemble}

Chen et al. (2012) again used the coordination properties of GSH and Cys. In their work, they formed a molecular ensemble in which cuprous ion quenches alizarin red dye. Upon addition of GSH, it chelates the Cu atoms and turn ON the emission of this dye (Figure 3D). The LOD was determined to be $2.3 \mathrm{~nm}$, which is very good considering the simplicity of the method. The test is also suitable for a colorimetric assay.

Molecular beacons are typically used to detect short sequences of complementary nucleic acids (Vet, Van der Rijt \& Blom, 2002), but they were adapted to detect GSH. They are composed of a single-stranded oligonucleotide with self-complementary $5^{\prime}$ and $3^{\prime}$ ends that can self-hybridize forming a structure known as hairpin (or stem loop). When a fluorescent dye is attached to one end and a suitable quencher is attached to the other end, when the molecule is hybridized, they come closer to each other, and the fluorescence is turned OFF. Xu and Hepel (2011) used a molecular beacon that has carboxy fluorescein dye, namely, 6-FAM at the 5' end and at the $3^{\prime}$ end a quencher 4-(4-dimethylaminophenylazo) benzoic acid (DABCYL). Near the end of the structure, there is a thymine-thymine mismatch. This mismatch is known to specifically form a stable complex with $\mathrm{Hg}^{2+}$ and can increase the $T_{\mathrm{m}}$ of the structure by approximately $10^{\circ} \mathrm{C}$. Glutathione and Cys can extract the $\mathrm{Hg}^{2+}$ ion from the mismatch $\mathrm{T}-\mathrm{Hg}^{2+}-\mathrm{T}$ complex, hence causing the stem-loop to change to the linear structure (Figure 3E). This will separate the fluorescent dye from the quencher, which allows emission. 


\subsubsection{Fluorescent dyes derivative and cell imaging}

This section discusses the recent advances in using old fluorescent probes in detecting GSH and how they could be chemically modified to improve certain functionality of the test. This could be achieved by adding chemical side chains that do not permanently deactivate the fluorophores. Moreover, they can improve cell membrane permeability or even help targeting a certain cellular component, improve specificity, or add switching properties to the molecule.

Chen et al. (2010) used nonfluorogenic organoselenium derivative of rhodamine (complex Rh-Se-2). It reacts with biothiols producing the high-quantum-yield rhodamine 110. This enabled the detection of small concentrations of GSH in a novel microchip electrophoresis device. They utilized the specific reaction of thiols to cleave the Se-N bond to detect biothiols in biosamples. They also included in the test another rhodamine dye derivative that is sensitive to $\mathrm{H}_{2} \mathrm{O}_{2}$. The device then separates and quantifies the two dyes according to their molecular charge using electrophoresis. The dye does not show specificity to GSH among other biothiols (Tang et al., 2009).

In order to improve the specificity of a rhodamine derivative dye to GSH, Yang et al. (2014) developed a rhodamine derivative dye that can differentiate between GSH and other thiolated amino acids Cys and Hcy. It was designed to emit at different wavelengths according to the nature of the thiolated analyte. This dye reacts with Cys and Hcy to produce deconjugated spirolactam ("quinone-phenol" rhodol structure) via a native chemical ligation (NCL) reaction. Upon photoexcitation, it leads to an excited-state intramolecular proton transfer (ESIPT) process that involves the proton of the phenolic hydroxyl group and the aromatic nitrogen in the benzothiazole ring as shown in the structure. On the other hand, the GSH can undergo the transthioesterification that deactivates the intramolecular photo-induced electron transfer (PET) process. This happens due to the electron withdrawing effect of the 4-nitrobenzene moiety, which produces an intense fluorescence enhancement at $587 \mathrm{~nm}$.

Cyanine dyes are well known for their strong fluorescence and biocompatibility. They were also chemically modified to specifically detect GSH among other biothiols and to image its cellular content using fluorescence microscopy. Yin et al. (2014) prepared two cyanine derivative dyes (CDDs) containing sulfonamide functional moiety located in the GSH reaction position of the dye. The sulfonamides are linked to the cyanine moiety through a piperazine bridge, which is cleaved in the presence of thiols to form free piperazine, simultaneously turning ON fluorescence. It showed the ability to permeate cell membrane. It was interesting that one probe was found to be selective to GSH, whereas the other probe did not show the same selectivity. Upon studying the structure using density function theory density functional theory calculations, the piperazine ring in the selective compound 2 exhibits chair conformation, whereas in the nonselective compound, it had a twisted boat conformation. This finding combined with the obvious structural difference between GSH and the other thiols could explain the differences observed in reactivities with the sulfonamide moiety in these fluorescent probes. Glutathione contains two amide units, and it has a long and flexible backbone compared to Cys and Hcy. That may enable intermolecular interactions with the probe. The team could successfully image the GSH-rich areas inside living cells. Also, they could monitor the level of GSH in mouse tissues such as liver, spleen, and kidneys.

BODIPY or boron-dipyrromethene dyes are attractive to use as fluorescent probes in biological systems, as they have relatively high molar absorption coefficients and quantum yields and narrow and high-intensity emission peaks (Leen et al., 2011). They are relatively inert in physiological conditions and less prone to photobleaching. Niu et al. (2012) report a specificity-enhanced fluorescent probe based on BODIPY. They synthesized a BODIPY derivative that is monochlorinated. This chlorine group can be rapidly replaced by thiolates of biothiols through thiol-halogen nucleophilic substitution. The amino groups of Cys/Hcy but not GSH can replace the thiolate to form amino-substituted BODIPY, which has much diminished fluorescence. So, the resulting compound after the reaction with Cys/Hcy will not be highly fluorescent, whereas the compound resulting from the reaction with GSH will be fluorescent.

Wei et al. (2013) prepared a fluorescent coumarin-based dye and attached a strong electron withdrawing group via a sulfonamide bond to form dinitrobenzenesulfonyl coumarin (DNBSQ). This group quenches the fluorescence via intramolecular charge transfer. The sulfonamide bond is cleaved in the presence of biothiols, which removes the quenching group and restores the intense fluorescence. This method is not specific for GSH, as it also responds to Cys. The probe was used successfully to image intracellular biothiols and demonstrated good cell permeability.

\subsubsection{Metal-ligand complexes}

Metal-ligand complexes have gained a great interest in the field of biosensing. Transition heavy metal complexes possess particular useful properties that makes them attractive in sensing applications (Taha et al., 2017; 
Elmalla \& Mansour, 2019; Mabrouk et al. 2019a; 2019b). For example, they have a long lifetime that allows their emission to be distinguished by fluorescent media using time-resolved luminescence spectroscopy (Terpetschnig et al., 1995). That is why the term "luminescence" is used to describe their mixed mode of emission rather than fluorescence or phosphorescence. They usually display large Stokes shifts, which advantageously do not allow self-quenching. It is quite easy to conjugate them with biomolecules. Also, their versatile photophysical properties can be easily tuned. Iridium, ruthenium, and osmium are among the most used metals in this type of probes.

Leung etal. (2013) used an iridium luminescent complex (luminescent iridium(III) complex $\left[\operatorname{Ir}(\mathrm{phq})_{2}(\mathrm{dpp})\right]^{+}$, where phq $=2$-phenylquinoline and dpp $=2,9$-diphenyl-1,10-phenanthroline) that works as a probe for DNA G-quadruplexes. When the quadruplex-probe complex is treated with a quencher like $\mathrm{Hg}^{2+}$ ion, the luminescence of the complex is greatly reduced. Biological thiols such as GSH and Cys can form a stable $\mathrm{Hg}^{2+}-\mathrm{S}$ adduct with the $\mathrm{Hg}^{2+}$ ions and sequester it, allowing the luminescence to be restored as depicted in Figure 3F.

\subsubsection{Surface-enhanced Raman scattering}

Surface-enhanced Raman scattering attracted increasing interest in different research fields because of its high sensitivity, selectivity, and extensive molecular information output. It is widely established that large SERS signal enhancement originates mainly from both an electromagnetic) mechanism and a charge-transfer mechanism. On the other hand, SERS suffers from poor reproducibility and low substrate stability, especially with substrates that depend on a localized surface plasmon resonance (LSPR). This could be addressed by using a well-fabricated nanostructured surface. Also, it suffers from low enhancement factor for the detections of biomolecules. However, SERS is widely used in surface analysis, biotechnology, environmental analysis, forensic science, and other fields. Only a few articles were published for the GSH determination using SERS. This is due to the low enhancement factor for GSH.

A novel SERS method called "heat-induced SERS sensing method" is developed by Huang et al. (Huang et al., 2009). A 60-nm silver nanoparticle colloidal suspension is used as a SERS substrate; before each SERS measurement, GSH mixed with the silver nanoparticles colloidal suspension is heated at 100 degrees for 3 min to cast a dry film of the SERS substrate layer. By optimizing different factors such as dropped sample volume, drying temperature, buffer concentration, and $\mathrm{pH}$ of the solution, highly enhanced GSH SERS spectra could be obtained, and quantitative determination of GSH was achieved by correlating the SERS intensity with GSH concentrations.

\subsubsection{Perspective of spectroscopic probes for $\mathrm{CSH}$}

Determination of GSH specifically by targeting organelles is an interesting new approach. One excellent example of targeting a cellular organelle and image GSH is the work of Lim et al. (2014). They designed a CDD in which they added a tunable lipophilic cation of a heptamethine unit. This unit functions as mitochondrial marker. Also, a nitroazo group was used as the GSH-specific reaction unit, as well as the fluorescence quencher. The initial nitroazo probe is not fluorescent, but upon reaction with GSH, the emission turned ON (at the NIR region, which lowers the background noise). The labile nitroazo aryl ether group reacts with GSH, Cys, and HCys, but only the GSH product is fluorescent. This may be because both Cys and Hcy form stable amino-substituted covalent complexes with the dye molecule through a sequential thiol substitution and rearrangement reaction.

Carbon dots are also gaining increasing interest and have been exploited for determination of GSH in a fetal calf serum sample (Wang et al., 2018). Oxidase mimics (Rao et al., 2018; Ge et al., 2019) are promising approaches for determination of GSH based on the ability of the latter to preclude the oxidases' activity, thus diminishing the formation of colored products from colorless precursors. This approach is expected to be extended in the future to make GSH detection easy and fast using a simple colorimeter.

\subsection{Technical aspects and artifacts in CSH assay methods}

The reported normal levels of GSH and GSSG in human plasma are highly variable, which could be due to possible artifacts in measurement procedures (Rossi et al., 2002; Hajdinák et al., 2019). Accurate determination of GSH/GSSG in biological samples requires precautions during collection, storage, preparation, and analysis. Blood samples must be collected in tubes prefilled with an anticoagulant (preferably tripotassium EDTA) and the derivatizing agent (usually NEM) (Giustarini et al., 2013). The sample should be slowly tilted for 1 min to 
ensure efficient mixing. Prolonged mixing should be avoided to evade GSH oxidation (Rossi et al., 2002). Addition of NEM as a derivatizing agent before sample acidification in the deproteinization step precludes GSH oxidation (Giustarini et al., 2003). Although several derivatizing agents have been used for GSH modification, NME is still widely used because of its ability to penetrate human cells and irreversibly block GSH reductase (Giustarini et al., 2016). In addition, the reaction of NEM with GSH is fast and does not require heating, and the alkylated derivative of GSH is stable against oxidation. This undesirable oxidation of GSH is a major source of assay errors because 1\% oxidation of GSH can induce 150\% bias in GSSG determination (Giustarini et al., 2016). The main limitation of NME is the low sensitivity, a problem that could be solved by using an additional derivatizing agent for fluorescence detection (Giustarini et al., 2003) or an MS detector that is inherently sensitive (Herzog et al., 2019). Sample storage is possible at $-80{ }^{\circ} \mathrm{C}$ for up to 3 months; otherwise, immediate sample preparation and analysis must be performed (Tipple \& Rogers, 2012). It should be noted that GSH measurements are highly affected by temperature, so all reagents need to be at ambient temperature before measurements (Haberhauer-Troyer et al., 2013).

Preparation of biological samples is mandatory to protect analytical instruments, improve method selectivity, and preconcentrate the analyte (Mansour \& Khairy, 2017). For plasma samples of GSH, sample preparation can be performed using protein precipitation, liquid-liquid extraction, or solid phase extraction (Huang et al., 2011a). Protein precipitation was carried out using water-miscible organic solvents (Methanol, acetonitrile) or mineral acids (TCA or perchloric acid). Best results were obtained when TCA was used because of minimal GSH loss (Rossi et al., 2002). At this stage, it is recommended to remove excess TCA and derivatizing agent residues using an immiscible solvent such as ether, ethyl acetate, or dichloromethane (DCM). In this case, DCM is preferred because of the high density, which makes DCM form a separate lower layer, and facilitates separation of the floating aqueous supernatant for GSH determination (Tietze, 1969).

Liquid-liquid extraction is another option for sample preparation, but the temperature used during the evaporation step increases the risk of GSH loss. Solid phase extraction is expensive, utilizing disposable cartilages and has the risk of cross contamination, if the cartilage was used for more than one sample. That may explain why protein precipitation is often preferred in GSH bioanalysis. The assay is usually performed by HPLC because of high selectivity and sensitivity, especially with MS detection. Special consideration should be given to the separation mode due to the high polarity of GSH; otherwise, it may elute unretained in RPLC or stick to the column in NPLC. Due to the chiral centers in GSH, chemical derivatization may form diastereomers, which can be separated in HPLC with regular columns. In this case, more than one peak could be obtained for GSH (Kuninori \& Nishiyama, 1985). The calibration curve should be constructed using one of the two peaks, which improves the method selectivity, if the other peak was overlapped with endogenous substances (Giustarini et al., 2003). Figure 4 summarizes the steps of GSH determination in blood and the essential precautions.

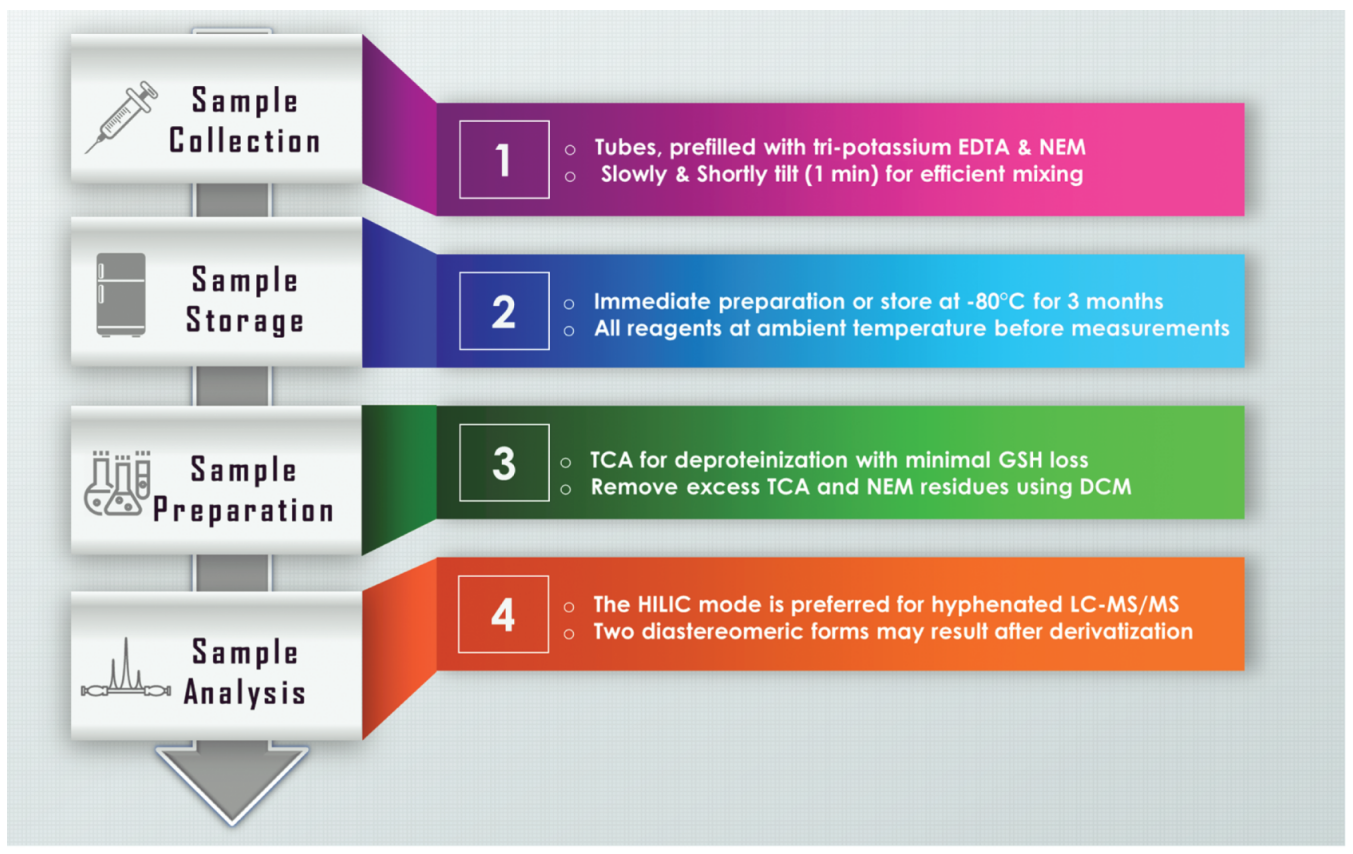

Figure 4: Procedures and precautions for GSH determination in blood. 


\section{Conclusion}

The recent methods used for determination of GSH in pharmaceutical and biological matrices were reviewed. The chromatographic methods using HPLC are widely applicable because of the instrument availability and the less complicated sample preparation procedures. Capillary electrophoresis offered the advantages of high selectivity and small required sample volume. Electrochemical methods are common because of the redox activity of GSH, and the research on chemical modifications of electrodes is ongoing. Nanotechnology has been exploited in both electrode modification and developing new sensors for GSH. These sensors have high selectivity and sensitivity, which makes them promising techniques and increase their chances to find a way to the market. All these methods and the methods to come will help gain a better understanding of the role of GSH in protection and in early diagnosis of different diseases.

\section{References}

Abolghasemi-Fakhri, Z.; Amjadi, M.; Manzoori, J. L. Exploring the behavior of gold nanostar@reduced graphene oxide composite in chemiluminescence: application to highly sensitive detection of glutathione. Spectrochim. Acta A 2019, 216, 85-90.

Alisik, M.; Neselioglu, S.; Erel, O. A colorimetric method to measure oxidized, reduced and total glutathione levels in erythrocytes. J. Lab. Med. 2019, 43, 269-277.

Amir, A. B.; Chobadi, S. Studies on oxidants and antioxidants with a brief glance at their relevance to the immune system. Life Sci. 2016, 146, 163-173.

Asimakopoulos, A. G.; Xue, J.; De Carvalho, B. P.; Iyer, A.; Abualnaja, K. O.; Yaghmoor, S. S.; Kumosani, T. A.; Kannan, K. Urinary biomarkers of exposure to 57 xenobiotics and its association with oxidative stress in a population in Jeddah, Saudi Arabia. Environ. Res. 2016, 150, 573-581.

Balabanova, D.; Remans, T.; Vassilev, A.; Cuypers, A.; Vangronsveld, J. Possible involvement of glutathione S-transferases in imazamox detoxification in an imidazolinone-resistant sunflower hybrid. J. Plant Physiol. 2018, 221, 62-65.

Banerjee, S.; Kar, S.; Perez, J. M.; Santra, S. Quantum dot-based OFF/ON probe for detection of glutathione. J. Phys. Chem. C 2009, 113, 96599663.

Bayram, B.; Rimbach, G.; Frank, J.; Esatbeyoglu, T. Rapid method for glutathione quantitation using high-performance liquid chromatography with coulometric electrochemical detection. J. Agr. Food Chem. 2014, 62, 402-408.

Benhar, M.; Shytaj, I. L.; Stamler, J. S.; Savarino, A. Dual targeting of the thioredoxin and glutathione systems in cancer and HIV. J. Clin. Invest. 2016, 126, 1630-1639.

Berenbaum, M. R.; Johnson, R. M. Xenobiotic detoxification pathways in honey bees. Curr. Opin. Insect Sci. 2015, 10, 51-58.

Bravo-Veyrat, S.; Hopfgartner, G. High-throughput liquid chromatography differential mobility spectrometry mass spectrometry for bioanalysis: determination of reduced and oxidized form of glutathione in human blood. Anal. Bioanal. Chem. 2018, 410, 7153-7161.

Cevasco, G.; Piątek, A. M.; Scapolla, C.; Thea, S. An improved method for simultaneous analysis of aminothiols in human plasma by highperformance liquid chromatography with fluorescence detection. J. Chromatogr. A 2010, 1217, 2158-2162.

Chang, C. W.; Tseng, W. L. Gold nanoparticle extraction followed by capillary electrophoresis to determine the total, free, and protein-bound aminothiols in plasma. Anal. Chem. 2010, 82, 2696-2702.

Chen, Y.; Dong, H.; Thompson, D. C.; Shertzer, H. G.; Nebert, D. W.; Vasiliou, V. Clutathione defense mechanism in liver injury: insights from animal models. Food Chem. Toxicol. 2013, 60, 38-44.

Chen, Z.; Li, Q.; Wang, X.; Wang, Z.; Zhang, R.; Yin, M.; Yin, L.; Xu, K.; Tang, B. Potent method for the simultaneous determination of glutathione and hydrogen peroxide in mitochondrial compartments of apoptotic cells with microchip electrophoresis-laser induced fluorescence. Anal. Chem. 2010, 82, 2006-2012.

Chen, Z.; Wang, Z.; Chen, J.; Chen, X. Alizarin red S/copper ion-based ensemble for fluorescence turn on detection of glutathione with tunable dynamic range. Biosens. Bioelectron. 2012, 38, 202-208.

Couto, N.; Wood, J.; Barber, ]. The role of glutathione reductase and related enzymes on cellular redox homoeostasis network. Free Radic. Biol. Med. 2016, 95, 27-42.

Crawford, L. A.; Weerapana, E. A tyrosine-reactive irreversible inhibitor for glutathione S-transferase Pi (CSTP1). Mol. Biosyst. 2016, 12, 17681771.

Dalle-Donne, I.; Rossi, R.; Colombo, G.; Giustarini, D.; Milzani, A. Protein S-glutathionylation: a regulatory device from bacteria to humans. Trends Biochem. Sci. 2009, 34, 85-96.

Elmalla, S. F.; Mansour, F. R. A simple innovative spectrofluorometric method for the determination of alendronate in bulk and in pharmaceutical tablets. Luminescence 2019, 34, 375-381.

Ensafi, A. A.; Bahrami, H.; Karimi-Maleh, H.; Mallakpour, S. Carbon paste electrode prepared from chemically modified multiwall carbon nanotubes for the voltammetric determination of isoprenaline in pharmaceutical and urine samples. Chinese ]. Catal. 2012a, 33, 19191926.

Ensafi, A. A.; Dadkhah-Tehrani, S.; Karimi-Maleh, H. Voltammetric determination of glutathione in haemolysed erythrocyte and tablet samples using modified-multiwall carbon nanotubes paste electrode. Drug Test. Anal. 2012b, 4, 978-985.

Ensafi, A. A.; Karimi-Maleh, H.; Mallakpour, S. A new strategy for the selective determination of glutathione in the presence of nicotinamide adenine dinucleotide (NADH) using a novel modified carbon nanotube paste electrode. Colloids Surf. B Biointerfaces 2013, 104, 186-193. 
Faber, S.; Fahrenholz, T.; Wolle, M. M.; Kern, J. C.; Pamuku, M.; Miller, L.; Jamrom, J.; Skip Kingston, H. M. Chronic exposure to xenobiotic pollution leads to significantly higher total glutathione and lower reduced to oxidized glutathione ratio in red blood cells of children with autism. Free Radic. Biol. Med. 2019, 134, 666-677.

Ferin, R.; Pavão, M. M. L.; Baptista, J. Methodology for a rapid and simultaneous determination of total cysteine, homocysteine, cysteinylglycine and glutathione in plasma by isocratic RP-HPLC. J. Chromatogr. B 2012, 911, 15-20.

Fracassetti, D.; Lawrence, N.; Tredoux, A. C. J. A.; Tirelli, A.; Nieuwoudt, H. H.; Du Toit, W. J. Quantification of glutathione, catechin and caffeic acid in grape juice and wine by a novel ultra-performance liquid chromatography method. Food Chem. 2011, 128, 1136-1142.

Cao, Y.; Li, Y.; Zou, X.; Huang, H.; Su, X. Highly sensitive and selective detection of biothiols using graphene oxide-based "molecular beacon"-like fluorescent probe. Anal. Chim. Acta 2012, 731, 68-74.

Ce, J.; Cai, R.; Chen, X.; Wu, Q.; Zhang, L.; Jiang, Y.; Cui, C.; Wan, S.; Tan, W. Facile approach to prepare HSA-templated $\mathrm{MnO}_{2}$ nanosheets as oxidase mimic for colorimetric detection of glutathione. Talanta 2019, 195, 40-45.

Giustarini, D.; Dalle-Donne, I.; Colombo, R.; Milzani, A.; Rossi, R. An improved HPLC measurement for CSH and CSSC in human blood. Free Radic. Biol. Med. 2003, 35, 1365-1372.

Giustarini, D.; Dalle-Donne, I.; Milzani, A.; Rossi, R. Detection of glutathione in whole blood after stabilization with N-ethylmaleimide. Anal. Biochem. 2011, 415, 81-83.

Giustarini, D.; Dalle-Donne, I.; Milzani, A.; Fanti, P.; Rossi, R. Analysis of CSH and CSSC after derivatization with N-ethylmaleimide. Nat. Protoc. 2013, 8, 1660-1669.

Giustarini, D.; Tsikas, D.; Colombo, G.; Milzani, A.; Dalle-Donne, I.; Fanti, P.; Rossi, R. Pitfalls in the analysis of the physiological antioxidant glutathione (CSH) and its disulfide (CSSC) in biological samples: an elephant in the room. J. Chromatogr. B Anal. Technol. Biomed. Life Sci. 2016, 1019, 21-28.

Giustarini, D.; Colombo, G.; Garavaglia, M. L.; Astori, E.; Portinaro, N. M.; Reggiani, F.; Badalamenti, S.; Aloisi, A. M.; Santucci, A.; Rossi, R.; Milzani, A.; Dalle-Donne, I. Assessment of glutathione/glutathione disulphide ratio and S-glutathionylated proteins in human blood, solid tissues, and cultured cells. Free Radic. Biol. Med. 2017, 112, 360-375.

Cłowacki, R.; Bald, E. Fully automated method for simultaneous determination of total cysteine, cysteinylglycine, glutathione and homocysteine in plasma by HPLC with UV absorbance detection. J. Chromatogr. B 2009, 877, 3400-3404.

Guo, X.-F.; Wang, H.; Guo, Y.-H.; Zhang, Z.-X.; Zhang, H.-S. Simultaneous analysis of plasma thiols by high-performance liquid chromatography with fluorescence detection using a new probe, 1,3,5,7-tetramethyl-8-phenyl-(4-iodoacetamido)difluoroboradiaza-s-indacene. J. Chromatogr. A 2009, 1216, 3874-3880.

Haberhauer-Troyer, C.; Delic, M.; Gasser, B.; Mattanovich, D.; Hann, S.; Koellensperger, C. Accurate quantification of the redox-sensitive CSH/CSSC ratios in the yeast Pichia pastoris by HILIC-MS/MS. Anal. Bioanal. Chem. 2013, 405, 2031-2039.

Hajdinák, P.; Czobor, Á.; Lőrincz, T.; Szarka, A. The problem of glutathione determination: a comparative study on the measurement of glutathione from plant cells. Period. Polytech-Chem. 2019, 63, 1-10.

Han, B.; Wang, E. Oligonucleotide-stabilized fluorescent silver nanoclusters for sensitive detection of biothiols in biological fluids. Biosens. Bioelectron. 2011, 26, 2585-2589.

Hanko, M.; Švorc, L'.; Planková, A.; Mikuš, P. Overview and recent advances in electrochemical sensing of glutathione - a review. Anal. Chim. Acta 2019, 1062, 1-27.

Harfield, J. C.; Batchelor-McAuley, C.; Compton, R. G. Electrochemical determination of glutathione: a review. Analyst 2012, 137, $2285-2296$.

Herzog, K.; IJlst, L.; van Cruchten, A. G.; van Roermund, C. W. T.; Kulik, W.; Wanders, R. J. A.; Waterham, H. R. An UPLC-MS/MS assay to measure glutathione as marker for oxidative stress in cultured cells. Metabolites 2019, 9, 45.

Hodáková, J.; Preisler, ].; Foret, F.; Kubáň, P. Sensitive determination of glutathione in biological samples by capillary electrophoresis with green (515 nm) laser-induced fluorescence detection. J. Chromatogr. A 2015, 1391, 102-108.

Honeychurch, K. C.; Hart, J. P. The chronoamperometric and voltammetric behaviour of glutathione at screen-printed carbon micro-band electrodes modified with cobalt phthalocyanine. Advances in Anal. Chem. 2012, 2, 46-52.

Huang, G. G.; Han, X. X.; Hossain, M. K.; Ozaki, Y. Development of a heat-induced surface-enhanced raman scattering sensing method for rapid detection of glutathione in aqueous solutions. Anal. Chem. 2009, 81, 5881-5888.

Huang, K.; Jing, Q.; Wei, C.; Wu, Y. Spectrofluorimetric determination of glutathione in human plasma by solid-phase extraction using graphene as adsorbent. Spectrochim. Acta A 2011a, 79, 1860-1865

Huang, Y. Q.; Ruan, G. D.; Liu, J. Q.; Gao, Q.; Feng, Y. Q. Use of isotope differential derivatization for simultaneous determination of thiols and oxidized thiols by liquid chromatography tandem mass spectrometry. Anal. Biochem. 2011b, 416, 159-166.

Ishida, A.; Fujii, M.; Fujimoto, T.; Sasaki, S.; Yanagisawa, I.; Tani, H.; Tokeshi, M. A portable liquid chromatograph with a battery-operated compact electroosmotic pump and a microfluidic chip device with a reversed phase packed column. Anal. Sci. 2015, 31, 1163-1169.

Janeš, L.; Lisjak, K.; Vanzo, A. Determination of glutathione content in grape juice and wine by high-performance liquid chromatography with fluorescence detection. Anal. Chim. Acta 2010, 674, 239-242.

Ji, Y.; Ma, Y.; Sun, X. Determination of glutathione in individual Ramos cells by capillary electrophoresis with electrochemiluminescence detection. Anal. Methods 2013, 5, 1542-1547.

Kamińska, A.; Olejarz, P.; Borowczyk, K.; Cłowacki, R.; Chwatko, G. Simultaneous determination of total homocysteine, cysteine, glutathione, and $\mathrm{N}$-acetylcysteine in brain homogenates by HPLC. J. Sep. Sci. 2018, 41, 3241-3249.

Karimi-Maleh, H.; Keyvanfard, M.; Alizad, K. Electrocatalytic determination of glutathione using multiwall carbon nanotubes paste electrode as a sensor and isoprenaline as a mediator. Int. J. Electrochem. Sci. 2012, 7, 6816-6830.

Karimi-Maleh, H.; Tahernejad-Javazmi, F.; Ensafi, A. A.; Moradi, R.; Mallakpour, S.; Beitollahi, H. A high sensitive biosensor based on FePt/CNTs nanocomposite/N-(4-hydroxyphenyl)-3,5-dinitrobenzamide modified carbon paste electrode for simultaneous determination of glutathione and piroxicam. Biosens. Bioelectron. 2014a, 60, 1-7.

Karimi-Maleh, H.; Tahernejad-Javazmi, F.; Cupta, V. K.; Ahmar, H.; Asadi, M. H. A novel biosensor for liquid phase determination of glutathione and amoxicillin in biological and pharmaceutical samples using a ZnO/CNTs nanocomposite/catechol derivative modified electrode. J. Mol. Liq. 2014b, 196, 258-263. 
Kern, J. K.; Geier, D. A.; Adams, J. B.; Garver, C. R.; Audhya, T.; Geier, M. R. A clinical trial of glutathione supplementation in autism spectrum disorders. Med. Sci. Monit. 2011, 17, 677-682.

Keyvanfard, M.; Karimi-Maleh, H.; Alizad, K. Multiwall carbon nanotube paste electrode with 3, 4-dihydroxy-cinnamic acid as mediator for the determination of glutathione in pharmaceutical and urine samples. Chinese J. Catal. 2013, 34, 1883-1889.

Khalikova, M. A.; Satinsky, D.; Solich, P.; Zinchenko, A. A.; Zhilyakova, E. T.; Novikov, O. O. A high-performance liquid chromatography method with pre-column derivatization for the simultaneous determination of reduced glutathione, carnosine and taurine. Anal. Methods 2014, $6,1475$.

Kominkova, M.; Horky, P.; Cernei, N.; Tmejova, K.; Ruttkay-Nedecky, B.; Guran, R.; Pohanka, M.; Zitka, O.; Adam, V.; Kizek, R. Optimization of the glutathione detection by high performance liquid chromatography with electrochemical detection in the brain and liver of rats fed with taurine. Int. J. Electrochem. Sci. 2015, 10, 1716-1727.

Kono, Y.; lizuka, H.; Isokawa, M.; Tsunoda, M.; Ichiba, H.; Sadamoto, K.; Fukushima, T. HPLC-fluorescence determination of thiol compounds in the serum of human male and female subjects using HILIC-mode column. Biomedical Chromatography, 2014, 28, 589-593.

Kowalska, K.; Zalewska, M.; Milnerowicz, H. The application of capillary electrophoresis in the determination of glutathione in healthy women's blood. J. Chromatogr. Sci. 2014, 53, 353-359.

Kubalczyk, P.; Bald, E. Analysis of orange juice for total cysteine and glutathione content by CZE with UV-absorption detection. Electrophoresis 2009, 30, 2280-2283.

Kuninori, T.; Nishiyama, J. Some properties of diastereomers formed in the reactions of tv-ethylmaleimide with biological thiols. Agric. Bio. Chem. 1985, 49, 2453-2454.

Kuśmierek, K.; Bald, E. Reversed-phase liquid chromatography method for the determination of total plasma thiols after derivatization with 1-benzyl-2-chloropyridinium bromide. Biomed. Chromatogr. 2009, 23, 770-775.

Kuśmierek, K.; Chwatko, G.; Cłowacki, R.; Kubalczyk, P.; Bald, E. Ultraviolet derivatization of low-molecular-mass thiols for high performance liquid chromatography and capillary electrophoresis analysis. J. Chromatogr. B 2011, 879, 1290-1307.

Lee, P. T.; Compton, R. G. Electrochemical detection of NADH, cysteine, or glutathione using a caffeic acid modified glassy carbon electrode. Electroanalysis 2013, 25, 1613-1620.

Lee, P. T.; Compton, R. G. Selective electrochemical detection of thiol biomarkers in saliva using multiwalled carbon nanotube screen-printed electrodes. Sens. Actuators B Chem. 2015a, 209, 983-988.

Lee, P. T.; Compton, R. G.; Selective thiol detection in authentic biological samples with the use of screen-printed electrodes. Anal. Sci. 2015b, 31, 685-691.

Lee, H.; Ko, E. H.; Lai, M.; Wei, N.; Balroop, J.; Kashem, Z.; Zhang, M. Delineating the relationships among the formation of reactive oxygen species, cell membrane instability and innate autoimmunity in intestinal reperfusion injury. Mol. Immunol. 2014a, 58, 151-159.

Lee, P.; Lowinsohn, D.; Compton, R.; Lee, P. T.; Lowinsohn, D.; Compton, R. G. The use of screen-printed electrodes in a proof of concept electrochemical estimation of homocysteine and glutathione in the presence of cysteine using catechol. Sensors 2014b, 14, $10395-10411$.

Lee, P. T.; Ward, K. R.; Tschulik, K.; Chapman, G.; Compton, R. G. Electrochemical detection of glutathione using a poly (caffeic acid) nanocarbon composite modified electrode. Electroanalysis 2014c, 26, 366-373.

Leen, V.; Miscoria, D.; Yin, S.; Filarowski, A.; Molisho Ngongo, J.; Van der Auweraer, M.; Boens, N.; Dehaen, W. 1,7-Disubstituted boron dipyrromethene (BODIPY) dyes: synthesis and spectroscopic properties. J. Org. Chem. 2011, 76, 8168-8176.

Leung, K.-H.; He, H.-Z.; Ma, V. P.-Y.; Chan, D. S.-H.; Leung, C.-H.; Ma, D.-L. A luminescent G-quadruplex switch-on probe for the highly selective and tunable detection of cysteine and glutathione. Chem. Commun. 2013, 49, 771-773.

$\mathrm{Li}, \mathrm{M}$.; Cheng, T.; Tseng, W. Nonionic surfactant-capped gold nanoparticles for selective enrichment of aminothiols prior to CE with UV absorption detection. Electrophoresis 2009, 30, 388-395.

Liedschulte, V.; Wachter, A.; Zhigang, A.; Rausch, T. Exploiting plants for glutathione (CSH) production: uncoupling CSH synthesis from cellular controls results in unprecedented CSH accumulation. Plant Biotechnol. J. 2010, 8, 807-820.

Lim, S.-Y.; Hong, K.-H.; Kim, D. I.; Kwon, H.; Kim, H.-J. Tunable heptamethine-azo dye conjugate as an NIR fluorescent probe for the selective detection of mitochondrial glutathione over cysteine and homocysteine. J. Am. Chem. Soc. 2014, 136, 7018-7025.

Liu, J.; Bao, C.; Zhong, X.; Zhao, C.; Zhu, L. Highly selective detection of glutathione using a quantum-dot-based OFF-ON fluorescent probe. Chem. Commun. 2010, 46, 2971.

Liu, X.; Zheng, P.; Zhao, X.; Zhang, Y.; Hu, C.; Li, J.; Zhao, J.; Zhou, J.; Xie, P.; Xu, G. Discovery and validation of plasma biomarkers for major depressive disorder classification based on liquid chromatography-mass spectrometry. J. Proteome Res. 2015, 14, 2322-2330.

Mabrouk, M.; Hammad, S. F.; Abdelaziz, M. A.; Mansour, F. R. Indirect spectrophotometric determination of ibandronate in pharmaceutical formulations via ligand exchange. Anal. Chem. Lett. 2019a, 9, 223-233.

Mabrouk, M.; Hammad, S. F.; Abdelaziz, M. A.; Mansour, F. R. Online postcolumn indirect detection for determination of ibandronate in pharmaceutical tablets by HPLC/DAD. J. Anal. Test. 2019b, 3, 322-330.

Mabrouk, M. M.; Soliman, S. M.; El-Agizy, H. M.; Mansour, F. R. A UPLC/DAD method for simultaneous determination of empagliflozin and three related substances in spiked human plasma. BMCChem. 2019c, 13, 83-91.

Mansour, F. R.; Danielson, N. D. Separation methods for captopril in pharmaceuticals and biological fluids. J. Sep. Sci. 2012, 35, $1213-1226$.

Mansour, F. R.; Danielson, N. D. Solidification of floating organic droplet in dispersive liquid-liquid microextraction as a green analytical tool. Talanta 2017, 170, 22-35.

Mansour, F. R.; Khairy, M. A. Pharmaceutical and biomedical applications of dispersive liquid-liquid microextraction. J. Chromatogr. B 2017, 1061-1062, 382-391.

Mansour, F. R.; Danielson, N. D. Solvent-terminated dispersive liquid-liquid microextraction: a tutorial. Anal. Chim. Acta 2018, $1016,1-11$.

Mansour, F. R.; Wei, W.; Danielson, N. D. Separation of carnitine and acylcarnitines in biological samples: a review. Biomed. Chromatogr. 2013, 27, 1339-1353.

Marchand, S.; de Revel, G. A HPLC fluorescence-based method for glutathione derivatives quantification in must and wine. Anal. Chim. Acta 2010, 660, 158-163. 
Mazloum-Ardakani, M.; Sheikh-Mohseni, M. A.; Mirjalili, B.-F. Selective and simultaneous voltammetric determination of glutathione, uric acid and penicillamine by a modified carbon nanotube paste electrode. Electroanalysis 2013, 25, 2021-2029.

Mendoza, J.; Garrido, T.; Riveros, R.; Parada, J. Rapid capillary electrophoresis analysis of glutathione and glutathione disulfide in roots and shoots of plants exposed to copper. Phytochem. Anal. 2009, 20, 114-119.

Michaelsen, J. J. T. J.; Dehnert, S.; Giustarini, D.; Beckmann, B.; Tsikas, D. HPLC analysis of human erythrocytic glutathione forms using OPA and $\mathrm{N}$-acetyl-cysteine ethyl ester: evidence for nitrite-induced CSH oxidation to CSSC. J. Chromatogr. B 2009, 877, 3405-3417.

Monostori, P.; Wittmann, G.; Karg, E.; Túri, S. Determination of glutathione and glutathione disulfide in biological samples: an in-depth review. J. Chromatogr. B 2009, 877, 3331-3346.

Moore, T.; Le, A.; Niemi, A. A.-K.; Kwan, T.; Cusmano-Ozog, K.; Enns, G. M.; Cowan, T. M. A new LC-MS/MS method for the clinical determination of reduced and oxidized glutathione from whole blood. J. Chromatogr. B 2013, 929, 51-55.

Moradi, R.; Sebt, S. A.; Karimi-Maleh, H.; Sadeghi, R.; Karimi, F.; Bahari, A.; Arabi, H. Synthesis and application of FePt/CNTs nanocomposite as a sensor and novel amide ligand as a mediator for simultaneous determination of glutathione, nicotinamide adenine dinucleotide and tryptophan. Phys. Chem. Chem. Phys. 2013, 15, 5888-5897.

Musenga, A.; Mandrioli, R.; Bonifazi, P.; Kenndler, E.; Pompei, A.; Raggi, M. A. Sensitive and selective determination of glutathione in probiotic bacteria by capillary electrophoresis-laser induced fluorescence. Anal. Bioanal. Chem. 2007, 387, 917-924.

Nakamura, T.; Hayashi, S. Enhancement of dye fluorescence by gold nanoparticles: analysis of particle size dependence. J Appl Phys. 2005, 44, 6833-6837.

Nesakumar, N.; Berchmans, S.; Alwarappan, S. Chemically modified carbon based electrodes for the detection of reduced glutathione. Sensor. Actuat. B Chem. 2018, 264, 448-466.

Niu, L.-Y.; Guan, Y.-S.; Chen, Y.-Z.; Wu, L.-Z.; Tung, C.-H.; Yang, Q.-Z. BODIPY-based ratiometric fluorescent sensor for highly selective detection of glutathione over cysteine and homocysteine. J. Am. Chem. Soc. 2012, 134, 18928-18931.

Özyürek, M.; Baki, S.; Cüngör, N.; Çelik, S. E.; Cüçlü, K.; Apak, R. Determination of biothiols by a novel on-line HPLC-DTNB assay with postcolumn detection. Anal. Chim. Acta 2012, 750, 173-181.

Panraksa, Y.; Siangproh, W.; Khampieng, T.; Chailapakul, O.; Apilux, A. Paper-based amperometric sensor for determination of acetylcholinesterase using screen-printed graphene electrode. Talanta 2018, 178, 1017-1023.

Pradhan, A.; Seena, S.; Schlosser, D.; Certh, K.; Helm, S.; Dobritzsch, M.; Krauss, G.-J.; Dobritzsch, D.; Pascoal, C.; Cássio, F. Fungi from metalpolluted streams may have high ability to cope with the oxidative stress induced by copper oxide nanoparticles. Environ. Toxicol. Chem. 2015, 34, 923-930.

Ragab, M. A. A.; El-Kimary, E. I. High performance liquid chromatography with photo diode array for separation and analysis of naproxen and esomeprazole in presence of their chiral impurities: enantiomeric purity determination in tablets. J. Chromatogr. A 2017, 1497, 110117.

Rao, H.; Zhu, X.; Wang, X.; Ding, F.; Liu, Y.; Zou, P.; Rao, H.; Zhao, Q.; Yang, L. Colorimetric determination of glutathione in human serum and cell lines by exploiting the peroxidase-like activity of CuS-polydopamine-Au composite. Anal. Bioanal. Chem. 2018, 410, 4805-4813.

Raoof, J. B. J.; Teymoori, N.; Khalilzadeh, M. A. M.; Ojani, R. A high sensitive electrochemical nanosensor for simultaneous determination of glutathione, NADH and folic acid. Mat. Sci. Eng. C 2015, 47, 77-84.

Robin, S.; Leveque, N.; Courderot-Masuyer, C.; Humbert, P. LC-MS determination of oxidized and reduced glutathione in human dermis: a microdialysis study. J. Chromatogr. B, 2011, 879, 3599-3606.

Rossi, R.; Milzani, A.; Dalle-Donne, I.; Ciustarini, D.; Lusini, L.; Colombo, R.; Di Simplicio, P. Blood glutathione disulfide: in vivo factor or in vitro artifact? Clin. Chem. 2002, 48, 742-753.

Sabiu, S.; O'Neill, F. H.; Ashafa, A. O. T. Membrane stabilization and detoxification of acetaminophen-mediated oxidative onslaughts in the kidneys of Wistar rats by standardized fraction of Zea mays L. (Poaceae), Stigma maydis. Evid. Based Complement. Alternat. Med.2016, 2016, 1-14.

Sánchez-Illana, Á.; Mayr, F.; Cuesta-Carcía, D.; Piñeiro-Ramos, J. D.; Cantarero, A.; Guardia, M. D. La.; Vento, M.; Lendl, B.; Quintás, G.; Kuligowski, J. On-capillary surface-enhanced raman spectroscopy: determination of glutathione in whole blood microsamples. Anal. Chem. 2018, 90, 9093-9100.

Schleicher, R. L.; Sternberg, M. R.; Looker, A. C.; Yetley, E. A.; Lacher, D. A.; Sempos, C. T.; Taylor, C. L.; Durazo-Arvizu, R. A.; Maw, K. L.; Chaudhary-Webb, M.; Johnson, C. L.; Pfeiffer, C. M. National estimates of serum total 25-hydroxyvitamin D and metabolite concentrations measured by liquid chromatography-tandem mass spectrometry in the US population during 2007-2010. J. Nutr. 2016, 146, 1051-1061.

Shabani-Nooshabadi, M.; Tahernejad-Javazmi, F. Rapid and fast strategy for the determination of glutathione in the presence of vitamin $\mathrm{B}_{6}$ in biological and pharmaceutical samples using a nanostructure based electrochemical sensor. RSC Adv. 2015, 5, 56255-56261.

Shahmiri, M. R.; Bahari, A.; Karimi-Maleh, H.; Hosseinzadeh, R.; Mirnia, N. Ethynylferrocene-NiO/MWCNT nanocomposite modified carbon paste electrode as a novel voltammetric sensor for simultaneous determination of glutathione and acetaminophen. Sensor. Actuat. B Chem. 2013, 177, 70-77.

Shen, C.-C.; Tseng, W.-L.; Hsieh, M.-M. Selective enrichment of aminothiols using polysorbate 20-capped gold nanoparticles followed by capillary electrophoresis with laser-induced fluorescence. J. Chromatogr. A 2009, 1216, 288-293.

Shen, C. C.; Tseng, W. L.; Hsieh, M. M. Selective extraction of thiol-containing peptides in seawater using Tween 20-capped gold nanoparticles followed by capillary electrophoresis with laser-induced fluorescence. J. Chromatogr. A 2012, 1220, 162-168.

Shi, Y.; Pan, Y.; Zhang, H.; Zhang, Z.; Li, M. M.-J.; Yi, C.; Yang, M. A dual-mode nanosensor based on carbon quantum dots and gold nanoparticles for discriminative detection of glutathione in human plasma. Biosens. Bioelectron. 2014, 56, 39-45.

Shishido, Y.; Tomoike, F.; Kimura, Y.; Kuwata, K.; Yano, T.; Fukui, K.; Fujikawa, H.; Sekido, Y.; Murakami-Tonami, Y.; Kameda, T.; Shuto, S.; Abe, H. A covalent G-site inhibitor for glutathione S-transferase Pi (CSTP $\left.{ }_{1-1}\right)$. Chem. Commun. 2017, 53, 11138-11141.

Siddiqui, M. R.; AlOthman, Z. A.; Rahman, N. Analytical techniques in pharmaceutical analysis: a review. Arab. J. Chem. 2017, 10, S1409-S1421.

Squellerio, I.; Caruso, D.; Porro, B.; Veglia, F.; Tremoli, E.; Cavalca, V. Direct glutathione quantification in human blood by LC-MS/MS: comparison with HPLC with electrochemical detection. J. Pharm. Biomed. Anal. 2012, 71, 111-118. 
Sun, X.; Heinrich, P.; Berger, R. S.; Oefner, P. J.; Dettmer, K. Quantification and 13C-Tracer analysis of total reduced glutathione by HPLCQTOFMS/MS. Anal. Chim. Acta 2019, 1080, 127-137.

Sutariya, V.; Wehrung, D.; Celdenhuys, W. J. Development and validation of a novel RP-HPLC method for the analysis of reduced glutathione. J. Chromatogr. Sci. 2012, 50, 271-276.

Taha, S.; Rafat, G.; Aboshosha, F.; Mansour, F. R. A simple homemade spectrophotometer. J. Anal. Chem. 2017, 72, $239-242$.

Tang, B.; Yin, L.; Wang, X.; Chen, Z.; Tong, L.; Xu, K. A fast-response, highly sensitive and specific organoselenium fluorescent probe for thiols and its application in bioimaging. Chem. Commun.2009, 5293-5295.

Terpetschnig, E.; Szmacinski, H.; Malak, H.; Lakowicz, J. R. Metal-ligand complexes as a new class of long-lived fluorophores for protein hydrodynamics. Biophys. J. 1995, 68, 342-350.

Tian, D.; Qian, Z.; Xia, Y.; Zhu, C. Cold nanocluster-based fluorescent probes for near-infrared and turn-on sensing of glutathione in living cells. Langmuir, 2012, 28, 3945-3951.

Tietze, F. Enzymic method for quantitative determination of nanogram amounts of total and oxidized glutathione: applications to mammalian blood and other tissues. Anal. Biochem. 1969, 27, 502-522.

Tipple, T. E.; Rogers, L. K. Methods for the determination of plasma or tissue glutathione levels. Methods Mol. Biol. 2012, 889, 315-324.

Tsardaka, E. C. E.; Zacharis, C. C. K.; Tzanavaras, P. P. D.; Zotou, A. Determination of glutathione in baker's yeast by capillary electrophoresis using methyl propiolate as derivatizing reagent. J. Chromatogr. A 2013, 1300, 204-208.

Turunc, E.; Karadeniz, H.; Armagan, G.; Erdem, A.; Yalcin, A. Electrochemical determination of glutathione in plasma at carbon nanotubes based screen printed electrodes. Comb. Chem. High Throughput Screen 2013, 16, 695-701.

Uehara, N.; Ookubo, K.; Shimizu, T. Colorimetric assay of glutathione based on the spontaneous disassembly of aggregated gold nanocomposites conjugated with water-soluble polymer. Langmuir, 2010, 26, 6818-6825.

Vet, J. A. M.; Van der Rijt, B. J. M.; Blom, H. J. Molecular beacons: colorful analysis of nucleic acids. Expert Rev. Mol. Diagn. 2002, 2, 77-86.

Wang, Y.; Xie, Y.; Bernier, M.; Wainer, I. W. I. Determination of free and protein-bound glutathione in HepC2 cells using capillary electrophoresis with laser-induced fluorescence detection. J. Chromatogr. A 2009, 1216, 3533-3537.

Wang, X.; Chi, D.; Song, D.; Su, G.; Li, L.; Shao, L. Quantification of glutathione in plasma samples by HPLC using 4-fluoro-7nitrobenzofurazan as a fluorescent labeling reagent. J. Chromatogr. Sci. 2012, 50, 119-122.

Wang, L.; Chen, H.; Wang, H.; Wang, F.; Kambam, S.; Wang, Y.; Zhao, W.; Chen, X. A fluorescent probe with high selectivity to glutathione over cysteine and homocysteine based on positive effect of carboxyl on nucleophilic substitution in CTAB. Sensor. Actuat. B Chem. 2014a, $192,708-713$.

Wang, X.; Wang, J.; Fu, H.; Liu, D.; Chen, Z. Determination of glutathione in single HepC2 cells by capillary electrophoresis with reduced graphene oxide modified microelectrode. Electrophoresis 2014b, 35, 3371-3378.

Wang, Q.; Pang, H.; Dong, Y.; Chi, Y.; Fu, F. Colorimetric determination of glutathione by using a nanohybrid composed of manganese dioxide and carbon dots. Microchim. Acta 2018, 185, 1-7.

Wei, M.; Yin, P.; Shen, Y.; Zhang, L.; Deng, J.; Xue, S.; Li, H.; Guo, B.; Zhang, Y.; Yao, S. A new turn-on fluorescent probe for selective detection of glutathione and cysteine in living cells. Chemical 2013, 49, 4640.

Wring, S. A.; Hart, J. P.; Bracey, L.; Birch, B. J. Development of screen-printed carbon electrodes, chemically modified with cobalt phthalocyanine, for electrochemical sensor applications. Anal. Chim. Acta 1990, 231, 203-212.

$\mathrm{Wu}$, J.; Ferrance, J. P.; Landers, J. P.; Weber, S. G. Integration of a precolumn fluorogenic reaction, separation, and detection of reduced glutathione. Anal. Chem. 2010, 82, 7267-7273.

Xu, H.; Hepel, M. “Molecular beacon"-based fluorescent assay for selective detection of glutathione and cysteine. Anal. Chem. 2011, 83, 813819.

Xue, R.; Wu, J.; Luo, X.; Gong, Y.; Huang, Y.; Shen, X.; Zhang, H.; Zhang, Y.; Huang, Z. Design, synthesis, and evaluation of diazeniumdiolatebased DNA cross-linking agents activatable by glutathione S-transferase. Org. Lett. 2016, 18, 5196-5199.

Yan, N.; Zhu, Z.; Ding, N.; Zhou, L.; Dong, Y.; Chen, X. In-line preconcentration of oxidized and reduced glutathione in capillary zone electrophoresis using transient isotachophoresis under strong counter-electroosmotic flow. J. Chromatogr. A 2009, 1216, 8665-8670.

Yang, X. X.-F.; Huang, Q.; Zhong, Y.; Li, Z.; Li, H.; Lowry, M.; Escobedo, J. O.; Strongin, R. M. A dual emission fluorescent probe enables simultaneous detection of glutathione and cysteine/homocysteine. Chemical 2014, 5, 2177-2183.

Yao, W.; Chang, L.; Yin, W.; Wang, T.; Yang, Y.; Yin, P.; Yang, M.; Ma, Y.; Qin, Y.; Ma, H. One immunoassay probe makes SERS and fluorescence two readout signals: rapid imaging and determination of intracellular glutathione levels. Spectrochim. Acta A 2019, 223, 117303.

Yin, J.; Kwon, Y.; Kim, D.; Lee, D.; Kim, G.; Hu, Y.; Ryu, J. H.; Yoon, J. Cyanine-Based fluorescent probe for highly selective detection of glutathione in cell cultures and live mouse tissues. J. Am. Chem. Soc. 2014, 136, 5351-5358.

Yuan, B.; Zeng, X.; Deng, D.; Xu, C.; Liu, L.; Zhang, J.; Gao, Y.; Pang, H. Electrochemical determination of glutathione based on an electrodeposited nickel oxide nanoparticles-modified glassy carbon electrode. Anal. Methods 2013, 5, 1779-1783.

Yuan, B.; Zhang, R.; Jiao, X.; Li, J.; Shi, H.; Zhang, D. Amperometric determination of reduced glutathione with a new Co-based metal-organic coordination polymer modified electrode. Electrochem. Commun. 2014, 40, 92-95.

Zhang, H.; Rinna, A. Glutathione: overview of its protective roles, measurement, and biosynthesis. Mol. Aspects Med. 2009, 30, 1-12.

Zhang, L. Y.; Sun, M. X. Fast determination of glutathione by capillary electrophoresis with fluorescence detection using $\beta$-cyclodextrin as modifier. Period. Polytech-Chem. 2009, 877, 4051-4054.

Zhang, Y.; Li, Y.; Yan, X.-P. Photoactivated CdTe/CdSe quantum dots as a near infrared fluorescent probe for detecting biothiols in biological fluids. Anal. Chem. 2009, 81, 5001-5007.

Zhang, W.; Li, P.; Ceng, Q.; Duan, Y.; Cuo, M.; Cao, Y. Simultaneous determination of glutathione, cysteine, homocysteine, and cysteinylglycine in biological fluids by ion-pairing high-performance liquid chromatography coupled with precolumn derivatization. J. Agr. Food Chem. 2014, 62, 5845-5852.

Zhang, Z.; Liu, T.; Wang, S.; Ma, J.; Zhou, T.; Wang, F.; Wang, X.; Zhang, G. DNA-templated gold nanocluster as a novel fluorometric sensor for glutathione determination. J. Photochem. Photobiol. A Chem. 2019, 370, 89-93. 